\title{
Estimation par une méthode variationnelle en élasticité des déformations lors du creusement d'un tunnel : application au calcul du tassement de surface
}

\author{
Estimates of ground movements due to tunneling \\ based on the variational principles of elasticity : \\ application to the determination of surface settlement
}

\author{
L. DORMIEUX, P. DE BUHAN \\ Laboratoire de Mécanique des Solides* \\ E. LECA \\ Laboratoire Central des Ponts et Chaussées" *
}

Rev. Franç. Géotech. n 59, pp. 15.32 (avril 1992)

\section{Résumé}

Le creusement d'un tunnel provoque une perturbation du massif encaissant qui se traduit le plus souvent par des venues de sol au niveau du tunnel et des tassements en surface. Le présent article est consacré à la quantification de ces phénomènes dans le cas du creusement au tunnelier, pour lequel les venues de sol se produisent principalement au front de taille et au niveau du vide interannulaire situé à l'arrière de la machine. Se plaçant dans le cadre de l'élasticité, on utilise le principe variationnel de minimum pour les contraintes pour établir une majoration analytique de ces venues de sol. Ces résultats sont appliqués au cas d'un matériau incompressible, pour lequel on propose une méthode d'estimation du tassement engendré en surface par le creusement d'un tunnel en régime permanent.

\footnotetext{
Abstract

The construction of a tunnel is usually associated with ground loss at the tunnel depth and settlements at the ground surface. The present paper deals with ground movement estimates for shield tunnels. In this case ground loss occurs mainly at the tunnel face and at the tail of the shield. The variationnal principles of elasticity are used to find an upper estimate of ground losses. The results are applied to the case of incompressibility, for which a method is proposed for estimating the surface settlement due to tunneling under steady state conditions.
} 


\section{INTRODUCTION}

L'utilisation croissante du sous-sol nécessite la réalisation de tunnels dans des conditions parfois difficiles, tant sur le plan géotechnique que du fait de contraintes d'environnement liées à l'existence d'ouvrages en surface. Les progrès accomplis sur le plan technologique durant les vingt dernières années ont permis de traiter en partie ce type de difficulté (HABIB, 1991). Parmi les innovations les plus marquantes, il faut citer la méthode du tunnelier et plus récemment, celle du tunnelier à front pressurisé. Cette technique consiste à effectuer la construction du tunnel à l'abri d'un cylindre d'acier, de section le plus souvent circulaire, destiné à retenir les parois du tunnel pendant le creusement. Ce type de machine assure généralement plusieurs fonctions : abattage du terrain au front de taille, évacuation des déblais, mise en place du soutènement à l'arrière du tunnelier. Les machines les plus perfectionnées visent également à stabiliser le front de taille pendant le creusement par application d'une pression de soutènement. Cette pression peut être obtenue par divers procédés : mise sous air comprimé de la galerie, confinement du terrain excavé dans la chambre d'abattage (tunnelier à pression de terre), application d'une pression de boue bentonitique. La propulsion de la machine est assurée par des vérins prenant appui sur la partie de l'ouvrage de soutènement déjà installée. Cette technique permet de réaliser des tunnels dans des terrains peu résistants.

Toutefois, le processus de creusement provoque une perturbation du massif encaissant et engendre des venues de sol au niveau du tunnel. Celles-ci résultent (1) de déplacements des terrains vers le front de taille, (2) de la perturbation du terrain situé au contact avec la jupe de la machine pendant la phase de propulsion, (3) d'éventuelles surcoupes ou déviations de la machine par rapport à une trajectoire rectiligne, et (4) de la convergence du terrain vers l'ouvrage au niveau du vide interannulaire, c'est-à-dire de l'espace compris entre le bord du terrain excavé et l'extrados du soutènement. Les méthodes récemment mises au point pour le creusement de tunnels en terrains meubles sont destinées à neutraliser ces venues de sol : le confinement du front de taille permet de retenir le terrain, voire de le repousser dans certains cas ; l'injection en continu d'un coulis sous pression à l'arrière de la machine permet, en théorie, de combler le vide interannulaire à mesure qu'il se forme. Dans la pratique, ces techniques connaissent des limites et des venues de sol sont fréquemment observées. Elles interviennent notamment au front de taille (en raison des fluctuations inévitables des pressions de soutènement) et au niveau du vide interannulaire.

Dans le premier cas, on tente de contenir en partie la venue de sol par l'application d'une pression de soutènement au front de taille. Dans le second, la venue de sol est essentiellement due à la différence entre le diamètre excavé et le diamètre revêtu. $\mathrm{Ce}$ vide interannulaire correspond à l'épaisseur de la jupe du bouclier augmentée d'un espace supplémentaire destiné à faciliter la mise en place de l'ouvrage à l'abri de la jupe du bouclier. Pendant la phase de progression de la machine, le vide laissé entre le terrain et l'anneau de soutènement tend à être comblé par la convergence du terrain vers l'ouvrage.

L'objet de la présente étude est d'estimer le volume de sol débouchant dans la galerie dans les deux situations qui viennent d'être évoquées en se plaçant, en première approximation, dans l'hypothèse où le comportement du matériau peut être modélisé par la théorie de l'élasticité linéaire.

Physiquement, il est clair que les tassements de surface résultant du creusement d'un tunnel à faible profondeur sont reliés aux venues de sol. En site urbain. ils doivent rester limités en sorte qu'aucun désordre n'apparaisse dans les constructions préexistantes. Le calcul des tassements apparait donc comme une application directe de celui des venues de sol.

Dans le cas général, il est malheureusement difficile de quantifier le lien qui existe entre venues de sol et tassements. Cependant, ce lien peut être précisé lorsque la compressibilité du sol dans lequel le forage est effectué peut être négligée. L'hypothèse d'incompressibilité peut être justifiée en particulier dans une analyse à court terme pour un massif de sol cohérent. Le calcul des venues de sol dans le cadre de la modélisation élastique basée sur les caractéristiques dites non drainées du matériau fournit alors une première estimation des tassements de surface. En d'autres termes, l'approche présentée ci-après fournit une évaluation du volume des venues de sol au niveau d'un tunnel quelle que soit la compressibilité du terrain. Mais lidentification avec les tassements de surface n'est possible, en toute rigueur, que dans le cas où $\nu=0,5$.

Dans le cadre de la modélisation élastique, les calculs numériques s'appuyent classiquement sur les deux principes variationnels de minimum. Parmi tous les champs de déplacements cinématiquement admissibles du problème, le champ solution minimise l'énergie potentielle. La méthode des éléments finis en déplacements en est une application bien connue. De manière analogue, parmi tous les champs de contraintes statiquement admissibles, le champ solution minimise l'énergie complémentaire.

On se propose de montrer dans la suite que ce dernier principe est le mieux adapté au problème posé et qu'il permet d'établir de façon simple (c'est-à-dire sans recourir à des calculs numériques souvent lourds) une estimation par excès du volume de sol débouchant dans la galerie.

\section{LE PROBLÈME POSÉ}

\subsection{Géométrie et matériau}

On considère un tunnel de forme cylindrique circulaire de diamètre $\mathrm{D}$ (rayon $\mathrm{R}$ ) foré horizontalement selon l'axe Ox dans un demi-espace limité supérieurement par le plan horizontal $y=C+R$. L'extension du tunnel est infinie dans la direction $\mathrm{x}<0$, le front de taille étant situé dans le plan $\mathrm{x}=0$ (fig. 1). Le comportement du matériau est modélisé par 


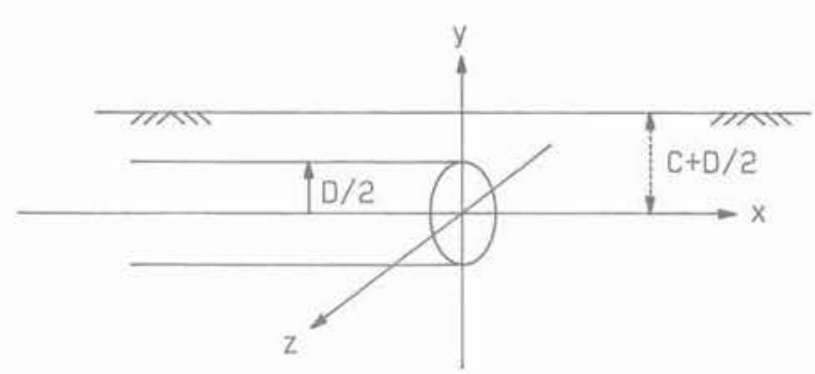

Fig. 1. - Schématisation de la géométrie.

Fig. 1. - Simplified geometry.

la loi élastique linéaire isotrope, caractérisée par un module d'Young $E$ et un coefficient de Poisson $\nu$ (ou de manière équivalente par les coefficients de Lamé $\lambda$ et $\mu$ ), supposés uniformes dans tout le massif. On envisage alors successivement les deux cas de chargement suivants.

\subsection{Cas de charge $n^{\circ} 1$}

A partir d'un état initial précontraint dû à l'action des forces de pesanteur, d'éventuelles surcharges de surface et de la pression de bouclier initiale $p_{0}(y)$, on étudie l'effet d'une diminution uniforme $-\delta \mathrm{p}$ de la pression de bouclier (fig. 2a). On suppose qu'il y a adhérence parfaite entre le revêtement latéral du tunnel, considéré comme rigide, et le massif (condition d'encastrement). En vertu du principe de superposition, le problème se ramène à l'étude d'une traction

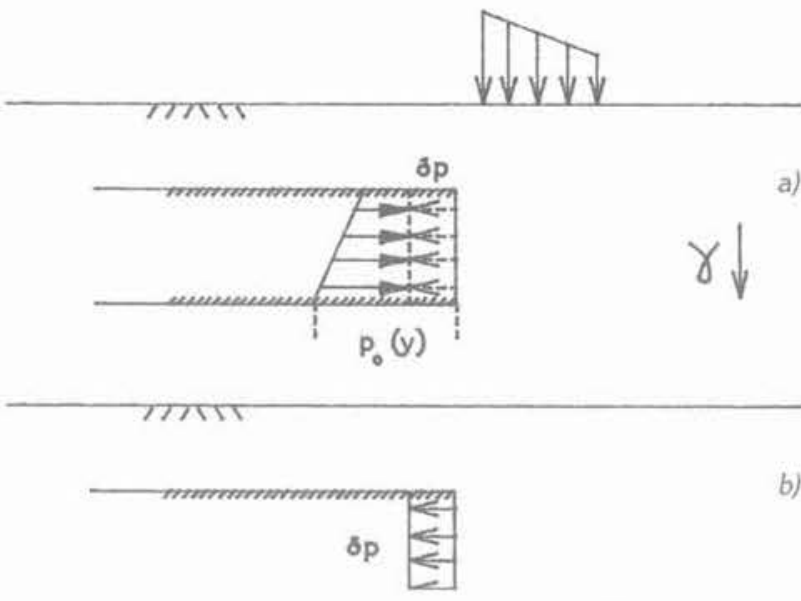

Fig. 2 a, b. - Cas de charge $n^{\circ} 1$

Fig. 2 a, b. - Loading case $n^{\circ} 1$.

uniforme - $\delta$ pe $\underline{x}_{\text {s }}$ sur le front de taille, dans un massif non pesant, sans surcharge de surface (fig. 2b).

\subsection{Cas de charge $\mathrm{n}^{\circ} 2$}

Il s'agit de modéliser l'effet d'un avancement du bouclier sur une distance L sur laquelle la mise en place du revêtement est imparfaite.

Le raisonnement consiste à considérer le système matériel obtenu en retranchant au massif la zone délimitée en pointillés sur la figure $3 a$, qui est excavée lors de lavancement L du bouclier. Dans un souci
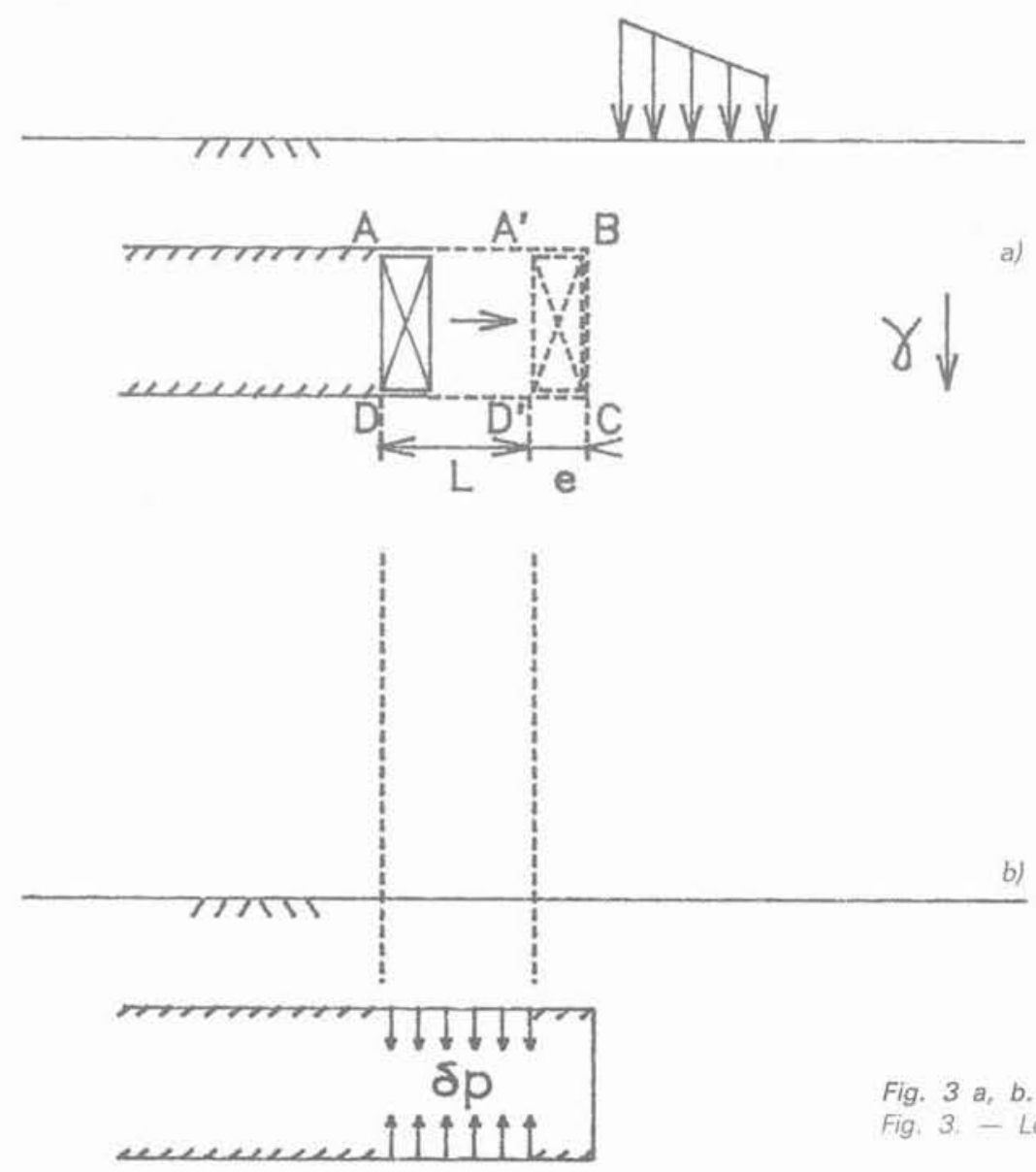

Fig. $3 a, b .-$ Cas de charge $n^{\circ} 2$. Fig. 3. - Loading case $n^{\circ} 2$. 
de clarté, cette figure ne respecte pas les proportions entre $\mathrm{L}$ et les dimensions usuelles d'un bouclier. Pour modéliser le forage, il s'agit de déterminer la variation des conditions aux limites prévalant sur la couronne cylindrique $\mathrm{ABCD}$ entre les instants où la face avant du bouclier est respectivement située dans les plans $\mathrm{x}=-\mathrm{L}$ puis $\mathrm{x}=0$. Cette tâche est rendue délicate par le fait qu'elle nécessite la connaissance de l'état de contraintes initial (avant forage) à droite du bouclier situé au départ dans le plan $\mathrm{x}=-\mathrm{L}$. Les hypothèses retenues sont les suivantes :

- les points du domaine matériel considéré situés sur la couronne cylindrique $\mathrm{A}^{\prime} \mathrm{BD}^{\prime} \mathrm{C}$ ne subissent aucun déplacement :

- la pression de bouclier est ajustée en sorte que le torseur des efforts agissant sur la face BC soit inchangé ;

- on admet que l'effet de décompression sur la couronne cylindrique $A A^{\prime} D^{\prime} D$ dû à l'avancement du bouclier peut être modélisé par une variation radiale $-\delta \mathrm{p} \underline{e}$, des forces surfaciques. Le choix du scalaire $\delta \mathrm{p}>0$ dépend de l'action de soutènement entreprise durant le forage entre les sections $x=-(L+e)$ et $\mathrm{x}=-e$.

En conclusion, Papplication du principe de superposition conduit à étudier un massif non pesant sans surcharge de surface dans lequel est forée une galerie soumise aux conditions aux limites suivantes (fig. 3b) :

- le front de taille situé dans le plan $\mathrm{x}=0$ est libre de contraintes ;

- la paroi cylindrique située entre les plans $x$ $=-(\mathrm{L}+e)$ et $\mathrm{x}=-e$ est soumise à une traction $-\delta \mathrm{p} \underline{e}_{\mathrm{r}}$;

- le déplacement est imposé nul sur les portions cylindriques $x<-(L+e)$ et $x \in]-e, 0[$.

\subsection{Lien entre volume de tassement et venue de sol dans le tunnel}

Soit $\xi$ le champ de déplacements dans le massif de sol pour l'un ou l'autre des deux cas de charges définis précédemment. Par définition le volume correspondant au tassement de surface global $\delta \mathrm{V}_{\mathrm{T}}$ est la quantité :

$$
\delta \mathrm{V}_{\mathrm{T}}=\int_{y=\mathrm{C}+\mathrm{R}}-\xi_{\mathrm{y}} \mathrm{da}
$$

Par ailleurs, la venue de sol $\delta V_{V}$ dans la galerie comporte deux termes, correspondant respectivement au front de taille $S_{t}$ et à la face latérale $S_{l}$ (fig. 4) :

$$
\delta \mathrm{V}_{\mathrm{V}}=\int_{\delta_{\ell}}-\xi_{\mathrm{r}} \mathrm{da}+\int_{\delta_{i}}-\xi_{\mathrm{x}} \mathrm{da}
$$

où $\xi_{\text {r }}$ désigne la composante radiale du déplacement dans le repère local de coordonnés cylindriques d'axe Ox. La convention de signe adoptée dans l'expression de $\delta \mathrm{V}_{\mathrm{V}}$ donnée ci-dessus confère, comme il se doit, un signe positif à un volume de sol débouchant à l'intérieur de la galerie.

Dans lhypothèse des petites perturbations qui est adoptée pour ce travail, la condition d'incompressibilité s'exprime par la relation div $\underline{\xi}=0$. On suppose de plus que le déplacement $\underline{\xi}$ s'annule au-delà d'une surface $\Sigma$ placée à une distance «suffisamment grande " du front de taille. On considère alors le domaine fermé $D$ (dont la frontière est tracée en traits renforcés sur la figure 4) qui est limité par la face latérale $S_{\ell}$ du tunnel, le front de taille $\mathcal{S}_{t}$, la surface $\Sigma$ et le plan $\mathrm{y}=\mathrm{C}+\mathrm{R}$. L'application du théorème de la divergence à ce domaine $D$ fournit :

$$
\begin{aligned}
& 0=\int_{D} \operatorname{div} \underline{\xi} \mathrm{d} \Omega=
\end{aligned}
$$

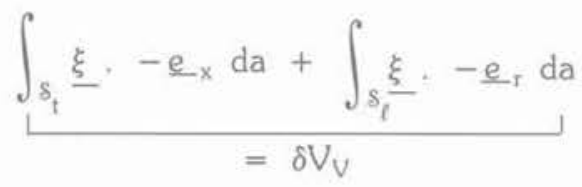

$$
\begin{aligned}
& +\underbrace{\int_{\Sigma} \underline{\xi}, \underline{\mathrm{n}} \mathrm{da}+\int_{y=\mathrm{C}_{\mathrm{C}}} \frac{\underline{\xi}}{L_{\mathrm{R}}} \cdot \underline{e}_{\mathrm{y}} \mathrm{da}}_{=0}
\end{aligned}
$$

où $n$ désigne la normale unitaire extérieure à $\partial D$. Compte tenu des définitions (1) et (2), l'égalité des volumes $\delta \mathrm{V}_{\mathrm{V}}$ et $\delta \mathrm{V}_{\mathrm{T}}$ en résulte immédiatement. Cette égalité ne doit pas masquer le fait que les déplace-

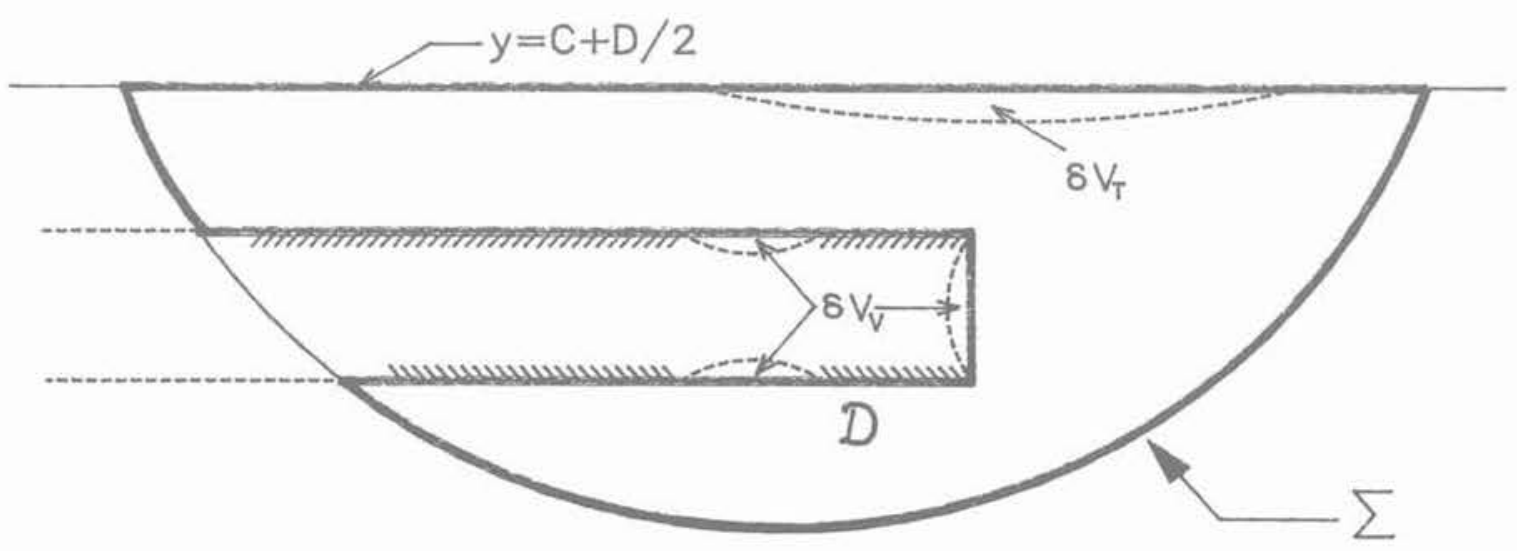

Fig. 4. - Venues de sol et tasseements dus au creusement d'un tunnel. Fig. 4. - Ground loss and settlement due to tunneling. 
ments au niveau du tunnel peuvent être sensiblement plus élevés que les tassements enregistrés en surface (HABIB, 1991).

\subsection{Calcul du tassement en régime permanent}

On se place dans les conditions du paragraphe cidessus pour lesquelles on vient de montrer que le volume de tassement peut être identifié à celui de la venue de sol. On se propose de montrer que l'analyse des cas de charges $n^{\circ} 1$ et 2 introduits précédemment permet également de prévoir le tassement de surface causé par le creusement d'un tunnel en régime permanent.

La progression du creusement d'un tunnel au bouclier est schématisée sur la figure $5 \mathrm{a}$. Le creusement du tunnel entraîne une perturbation du massif qui se traduit en surface par une cuvette de tassement. En régime permanent, la section de la cuvette de tassement par un plan longitudinal peut être représentée par une courbe qui s'amorce à l'avant du front de taille et se stabilise assez rapidement derrière la queue du bouclier. Chaque phase de creusement se traduit par lavancement de la machine sur une longueur $L$ d'environ $1 \mathrm{~m}$, correspondant à la longueur de l'anneau de soutènement installé à l'arrière de la machine. Il en résulte l'excavation d'un noyau de sol sensible. ment cylindrique (partie en hachures espacées sur la figure 5a). En faisant l'hypothèse d'une répétition parfaite du processus de creusement à chaque étape, l'excavation de ce noyau de sol se traduit en surface par une translation de longueur $\mathrm{L}$ du profil de tassement selon le sens de la progression du front de taille. Par conséquent, chaque étape de creusement provoque en surface un accroissement $\delta \mathrm{V}_{\mathrm{T}}$ du volume de tassement (hachures fines sur la figure 5a), obtenu par différence entre le profil de tassement final et celui de l'étape précédente.

Ainsi, pour une longueur creusée totale de $\mathrm{x}$, le volume de la dépression (ou tassement) de surface peut se mettre sous la forme suivante:

$$
V_{T}(x)=(x-a) S_{T}+V_{a}
$$

où $\mathrm{S}_{\mathrm{T}}$ représente le volume de tassement par unité de longueur dans la partie stabilisée, et $V_{a}$ désigne
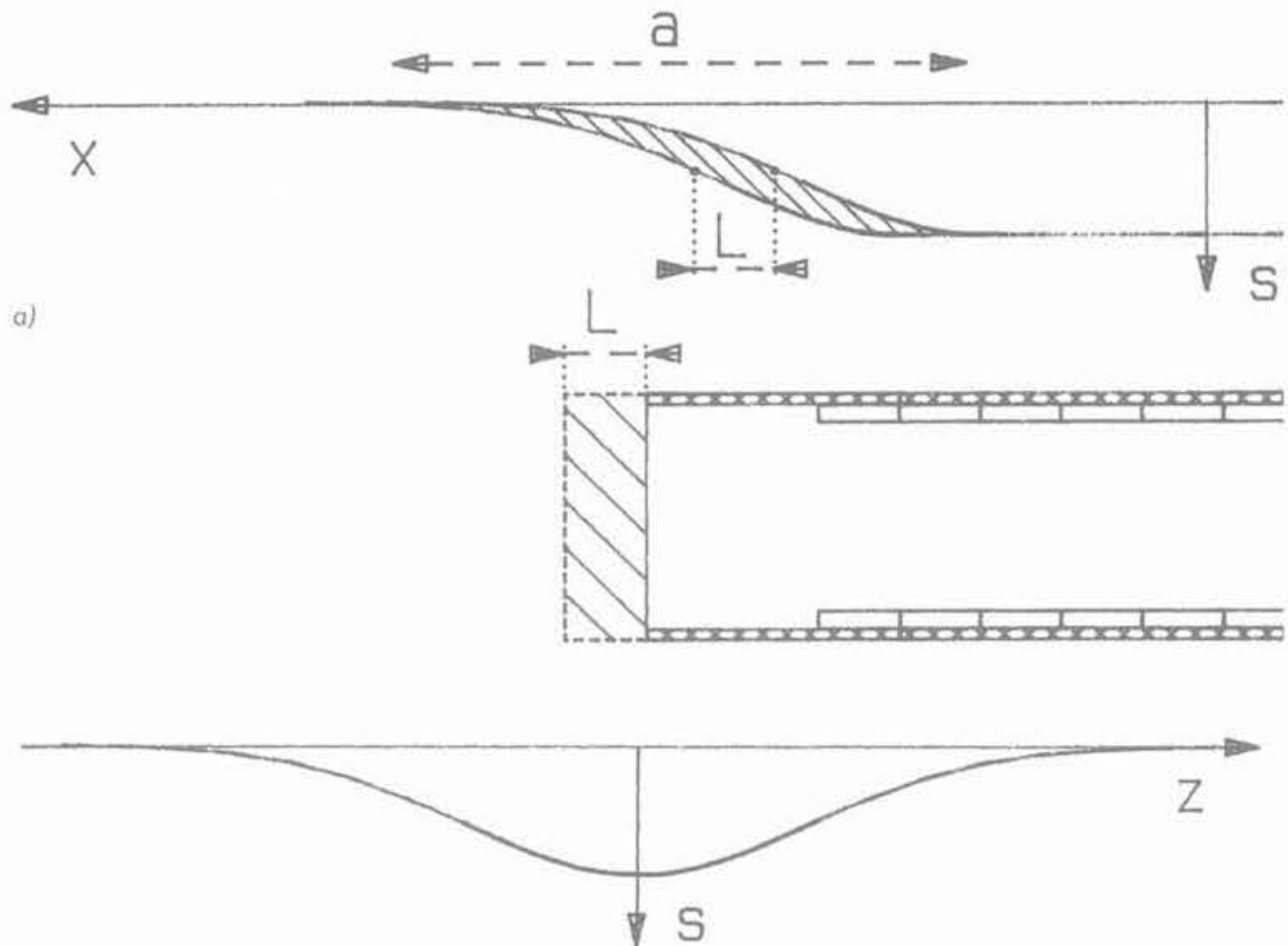

b)

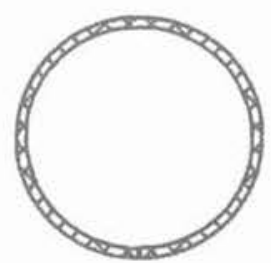

Fig. 5. - Profils de tassement de surface : $5 a$ : section longitudiale - $5 b$ : section transversale. Fig. 5. - Surface settlements profiles: $5 a$ : longitudinal section - 5 b: cross section. 
le volume de tassement de la partie située dans la zone d'influence directe du front de taille, d'étendue a. La progression de la machine sur une longueur L se traduit par une nouvelle cuvette de tassement de volume total

$$
V_{T}(x+L)=(x+L-a) S_{T}+V_{a}
$$

Le tassement $\delta \mathrm{V}_{\mathrm{T}}$ induit par l'avancée $\mathrm{L}$ du bouclier vaut donc:

$$
\delta \mathrm{V}_{\mathrm{T}}=\mathrm{V}_{\mathrm{T}}(\mathrm{x}+\mathrm{L})-\mathrm{V}_{\mathrm{T}}(\mathrm{x})=\mathrm{S}_{\mathrm{T}} \mathrm{L}
$$

Cette égalité confère une deuxième signification à la grandeur $\mathrm{S}_{\mathrm{T}}$ qui apparait ici comme le volume de tassement généré par le creusement de l'unité de longueur de tunnel.

En limitant la prise en compte du phénomène de venue de sol aux deux manifestations principales évoquées précédemment, le volume de tassement $S_{T}$ par unité de longueur creusée se met sous la forme suivante:

$$
\mathrm{S}_{\mathrm{T}}=\left(\delta \mathrm{V}_{1}+\delta \mathrm{V}_{2}\right) / \mathrm{L}
$$

où $\delta \mathrm{V}_{1}$ désigne le volume de tassement résultant des venues de sol au front de taille et $\delta \mathrm{V}_{2}$ celui qui résulte des mouvements de terrain dans le vide interannulaire. Plus précisément, soit $\delta \mathrm{p}_{1}$ l'écart moyen entre la contrainte normale s'exerçant sur le plan vertical situé à la distance $\mathrm{L}$ en amont du front de taille et la pression au front de taille. Le volume $\delta V_{1}$ peut être, en première approximation *, identifié à la venue de sol correspondant à une variation $\delta \mathrm{p}_{1}$ de la pression au front de taille dans le cas de charge $n^{\circ} 1$. Par définition, $\delta \mathrm{p}_{1}$ dépend à la fois de l'état de contraintes dans le terrain en avant du front de taille et de la valeur de la pression exercée au front de taille. De même, $\delta V_{2}$ représente la venue de sol résultant d'une décompression moyenne $\delta \mathrm{p}_{2}$ sur une longueur $\mathrm{L}$ à l'arrière de la machine au cours d'un avancement du bouclier sur cette même longueur (cas de charge $n^{\circ} 2$ ). La dépression $\delta \mathrm{p}_{2}$ dépend de l'état de contrainte dans le terrain avant décompression et de la pression utilisée pour l'injection du vide interannulaire.

Il reste à relier $\mathrm{S}_{\mathrm{T}}$ au tassement proprement dit. Dans un plan perpendiculaire à l'axe du tunnel, le profil de tassement observé (PECK, 1969) se présente généralement sous la forme d'une courbe de Gauss inversée (fig. 5b) d'équation :

$$
\mathrm{s}(\mathrm{z})=\mathrm{s}_{\max } \exp \left(-\mathrm{z}^{2} / 2 \mathrm{i}^{2}\right)
$$

où $\mathrm{s}_{\max }$ représente la valeur maximale du tassement. généralement observée au-dessus de l'axe du tunnel $(\mathrm{z}=0)$ et où i représente la distance du plan médian au point d'inflexion de la courbe de Gauss et caractérise l'étendue latérale du profil de tassement.

Si l'on fait l'hypothèse couramment admise dans la pratique que le tassement de surface peut effectivement être décrit par une relation du type donné en (4), la détermination du profil de tassement se ramène

\footnotetext{
- La superposition des cas de charge $n^{\circ} 1$ et $n^{\circ} 2$ pour simuler le creusement n'est pas totalement rigoureuse en raison de la nature des conditions aux limites sur la face latérale dans le cas de charge $n^{\circ} 1$
}

à l'estimation de $\mathrm{s}_{\max }$ et de $\mathrm{i}$, ou de manière équivalente, de $\mathrm{S}_{\mathrm{T}}$ et de $\mathrm{i}$. En effet, on a classiquement :

$$
\mathrm{S}_{\mathrm{T}}=\sqrt{2 \pi} \mathrm{s}_{\max } \mathrm{i} \cong 2,5 \mathrm{~s}_{\max } \mathrm{i}
$$

Des observations effectuées sur de nombreux chantiers (PECK, 1969 ; CORDING et HANSMIRE, 1975 ; ATTEWELL, 1977 ; CLOUGH et SCHMIDT, 1981) montrent que le paramètre i peut être évalué à partir de la connaissance de la nature du terrain encaissant et de la profondeur $\mathrm{H}=\mathrm{C}+\mathrm{D} 2$ du tunnel. S'agissant des ouvrages creusés en terrain argileux, on peut admettre en première approximation que $\mathrm{i} \cong \mathrm{H} / 2$.

Ainsi, l'approche proposée ci-après qui est consacrée aux venues de sol dans les cas de charge $n^{\circ} 1$ et $n^{\circ} 2$ permet en outre une estimation complète du profil des tassements de surface provoqués par le creusement d'un tunnel. Elle est particulièrement bien adaptée au cas des argiles dans le cadre d'une analyse du tassement à court terme.

\section{L'APPROCHE RETENUE}

On note U ( $\left.\xi^{\prime}\right)$ l'énergie potentielle d'un champ de déplacements $\bar{\xi}$ cinématiquement admissible (c.a.) et $\mathrm{C}(\underline{\underline{g}})$ l'énergie complémentaire d'un champ de contraintes $\underline{\underline{o}}$ statiquement admissible (s.a.). Les principes variationnels énoncés dans l'introduction s'expriment alors par les inégalités suivantes (voir par exemple SALENÇON, 1988) :

$$
\begin{aligned}
& (\forall \underline{\xi}(\text { c.a. }))(\forall \underline{\underline{\sigma}}(\text { s.a.)) } \\
& -U\left(\underline{\xi^{\prime}}\right) \leq-U(\underline{\xi})=C(\underline{\underline{\sigma}}) \leq C\left(\underline{\underline{\sigma^{\prime}}}\right)
\end{aligned}
$$

où $(\underline{\underline{\sigma}}, \underline{\xi})$ désigne le couple solution du problème d'élasticité posé. Il convient d'abord de préciser la structure des deux fonctionnelles énergétiques introduites dans (6) pour les problèmes considérés. L'énergie potentielle $U\left(\xi^{\prime}\right)$ prend la forme suivante:

$$
U\left(\xi^{\prime}\right)=W\left(\xi^{\prime}\right)-\Phi\left(\xi^{\prime}\right)
$$

où $W(\xi)$ représente l'énergie élastique dans le champ de déformations $\epsilon^{\prime}$ associé à ' $\xi$ et où $\Phi(\xi ')$ intègre les données en efforts du chargement considéré. Plus précisément, on aura :

$$
\left.W\left(\underline{\xi^{\prime}}\right)=\int_{\Omega} \frac{\lambda}{2}\left(\operatorname{tr} \stackrel{\epsilon^{\prime}}{=}\right)^{2}+\mu \operatorname{tr}{\left.\stackrel{\left(\epsilon^{\prime}\right.}{=}\right)}^{2}\right) d \Omega
$$

et respectivement pour les deux chargements :

- cas de charge $n^{\circ} 1$ :

$$
\Phi \underline{(\underline{\xi})}=\int_{\substack{x=0 \\ r<R}}-\delta \mathrm{p} \underline{e_{x}} \cdot \underline{\xi^{\prime}} \operatorname{rdrd} \theta
$$

- cas de charge $n^{\circ} 2$

$$
\Phi\left(\underline{\left.\xi^{\prime}\right)}=\int_{-(\mathrm{L}+\mathrm{e})}^{-e} \mathrm{dx} \int-\delta \mathrm{p} \underline{e_{r}} \cdot \underline{\xi^{\prime}} \operatorname{Rd} \mathrm{R}\right.
$$


De même, l'énergie complémentaire se décompose sous la forme suivante:

$$
C(\underline{\underline{\underline{\sigma}}})=W^{*}\left(\underline{\underline{\sigma^{\prime}}}\right)-\Phi^{*}\left(\underline{\underline{\underline{\sigma^{\prime}}}}\right)
$$

où $W^{*}(\underline{\underline{\sigma}})$ représente l'énergie élastique dans le champ de contraintes $\underline{\sigma}^{\prime}$ et où $\Phi^{*}\left(\underline{\sigma^{\prime}}\right)$ intègre les données en déplacements du chargement considéré. Dans les deux cas de charge considérés, les déplacements aux limites du domaine sont nuls là où ils sont imposés. La fonctionnelle $\Phi^{*}(\underline{\underline{\sigma}})$ est donc elle-même nulle. L'énergie élastique de contraintes $W^{*}\left(\underline{\underline{\sigma^{\prime}}}\right)$ a la forme suivante :

$W^{*}(\underline{\underline{\sigma}})=\frac{1}{2} \int_{\Omega} \frac{1+\nu}{\mathrm{E}} \operatorname{tr}\left(\underline{\underline{\sigma}}^{\prime 2}\right)-\frac{\nu}{\mathrm{E}}\left(\operatorname{tr} \underline{\underline{\sigma^{\prime}}}\right)^{2} \mathrm{~d} \Omega$

Le théorème de Clapeyron établit alors pour le couple solution $\underline{\underline{\sigma}}, \underline{\xi})$ les égalités suivantes :

$W^{*}(\underline{\underline{\sigma}})=W(\underline{\xi})=\frac{1}{2}\left(\Phi^{*}(\underline{\underline{\sigma}})+\Phi(\underline{\underline{\xi}})\right)$

d'où, d'après (10) :

$$
C(\underline{\underline{\sigma}})=W^{*}(\underline{\underline{\sigma}})=W(\underline{\underline{\xi}})=\frac{1}{2} \Phi(\underline{\underline{\xi}})
$$

puisque l'on a $\Phi^{*}(\underline{\sigma})=0$ dans les deux cas de chargement considérés. Il vient donc, dans le cas de charge $\mathrm{n}^{\circ} 1$ :

$$
C(\underline{\underline{\sigma}})=-\frac{1}{2} \int_{\substack{x=0 \\ r<R}} \delta \mathrm{p} \underline{\underline{e}} \times \cdot \underline{\xi} \operatorname{rdrd} \theta=\frac{1}{2} \delta V_{1} \delta \mathrm{p}
$$

où $\delta \mathrm{V}_{1}$ représente la venue de sol au niveau du front de taille, et dans le cas de charge $n^{\circ} 2$ :

$$
\begin{aligned}
& C(\underline{\underline{\sigma}})=-\frac{1}{2} \int_{-(L+e)}^{-\mathrm{e}} \mathrm{dx} \int_{0}^{2 \pi} \delta \mathrm{p} \underline{e_{r}}, \underline{\xi} \mathrm{Rd} \theta \\
& =\frac{1}{2} \delta \mathrm{V}_{2} \delta \mathrm{p}
\end{aligned}
$$

$\delta \mathrm{V}_{2}$ désignant la venue de sol au niveau de la couronne cylindrique décomprimée en arrière du front de taille.

La double inégalité (6) s'écrit en définitive :

$$
\begin{aligned}
& \left(\forall \underline { \sigma ^ { \prime } } \text { s.a.) } \left(\forall \underline{\xi^{\prime}}\right.\right. \text { c.a.) } \\
& -2 U\left(\underline{\xi^{\prime}}\right) / \delta \mathrm{p} \leq \delta \mathrm{V}_{\mathrm{f}} \leq 2 \mathrm{~W}^{*}\left(\underline{\underline{\sigma^{\prime}}}\right) / \delta \mathrm{p}
\end{aligned}
$$

Ainsi, l'utilisation conjointe des principes variationnels fournit un encadrement du volume de sol débouchant dans la galerie, pour chacun des deux cas de charges examinés. En général, c'est bien une estimation par excès de ce volume qui sera préférable, si l'on se place du point de vue de la sécurité. L'utilisation du principe de minimun pour les contraintes est donc particulièrement bien adaptée au problème posé.

Tout choix d'un champ de contraintes $\sigma^{\prime}$ s.a. pour le problème $\mathrm{n}^{\circ} \mathrm{i}$ tel que l'énergie élastique $\mathrm{W}^{*}(\underline{\underline{\underline{\sigma}}})$ reste bornée fournit a priori un majorant de la venue de sol $\delta \mathrm{V}_{i}$. On se propose à présent d'établir par ce procédé quelques résultats pratiques par voie analytique.

\section{APPLICATIONS DU PRINCIPE DE MINIMUM POUR LES CONTRAINTES}

\subsection{Cas de charge $n^{\circ} 1$}

\subsubsection{Champ en coordonnées sphériques}

Une première idée consiste à exploiter la solution en contraintes du problème de la sphère creuse élastique sous pression. Plus précisément, on considère les sphères $S_{R}$ et $S_{X}$ de centre $O$ et de rayons respectifs $\mathrm{R}$ et $\mathrm{X} \in \mathrm{]R}, \mathrm{C}+\mathrm{R}[$. On adopte le système de coordonnées sphériques $r, \theta, \varphi$, où le rayon $r$ est compté à partir de $\mathrm{O}$ et l'angle $\theta$ à partir de l'axe $\mathrm{Ox}$. Un champ de contraintes $\underline{\sigma}$ statiquement admissible avec $\delta$ p est défini de la manière suivante (fig. 6) : - dans la partie du massif située à l'intérieur de $S_{\mathrm{R}}$, notée $(\alpha)$ dans la suite, et définie par les conditions $r \in[0, \mathrm{R}], \quad \theta \in\left[0, \frac{\pi}{2}\right], \varphi \in[0,2 \pi], \underline{\sigma}=$ est un champ constant de traction isotrope égal à $\delta \mathrm{p} 1$ :

- dans la partie $(\beta)$ du massif comprise entre les rayons $\mathrm{r}=\mathrm{R}$ et $\mathrm{r}=\mathrm{X}, \sigma^{\prime}$ coïncide avec la solution de la sphère creuse soumise à une traction $-\delta$ p $\underline{e}$, sur la paroi de sa cavité interne $(r=R)$ et libre de contraintes sur sa face externe $(r=X)$, c'està-dire :

$\sigma_{n}^{\prime}=A+2 B / r^{3} \quad \sigma_{\theta \theta}^{\prime}=\sigma_{\varphi \varphi}^{\prime}=A-B / r^{3}$

où l'on a posé, notant $\rho_{\mathrm{x}}$ le rapport $\mathrm{X} / \mathrm{R}$ :

$$
\begin{aligned}
& A=-\delta \mathrm{p} /\left(\rho_{\mathrm{x}}^{3}-1\right) \\
& \mathrm{B}=\frac{1}{2} \delta \mathrm{pR}^{3} \rho_{\mathrm{x}}^{3} /\left(\rho_{\mathrm{x}}^{3}-1\right)
\end{aligned}
$$

- le champ $\underline{\underline{\sigma}}$ est nul en dehors de la sphère $S_{X}$.

Le rayon $X$ de la sphère extérieure décrivant le segment ]R, C + R], on dispose ainsi d'une classe à un paramètre de champs de contraintes s.a. pour le problème $n^{0} 1$. Pour optimiser le choix de X et obtenir le plus petit majorant de $\delta V_{1}$ dans la classe examinée, il reste à déterminer l'énergie élastique de contraintes correspondante.

La contribution du domaine $(\alpha)$ dans lequel $\sigma$ est le champ de contraintes sphériques $\delta \mathrm{p} 1$ résulte immédiatement de (11) :

$$
\begin{aligned}
& W_{\alpha}^{*}=\frac{3(1-2 \nu)}{2 E}(\delta p)^{2} \int_{(\alpha)} d \Omega \\
& =(1-2 \nu) \frac{\pi R^{3}(\delta p)^{2}}{E}
\end{aligned}
$$




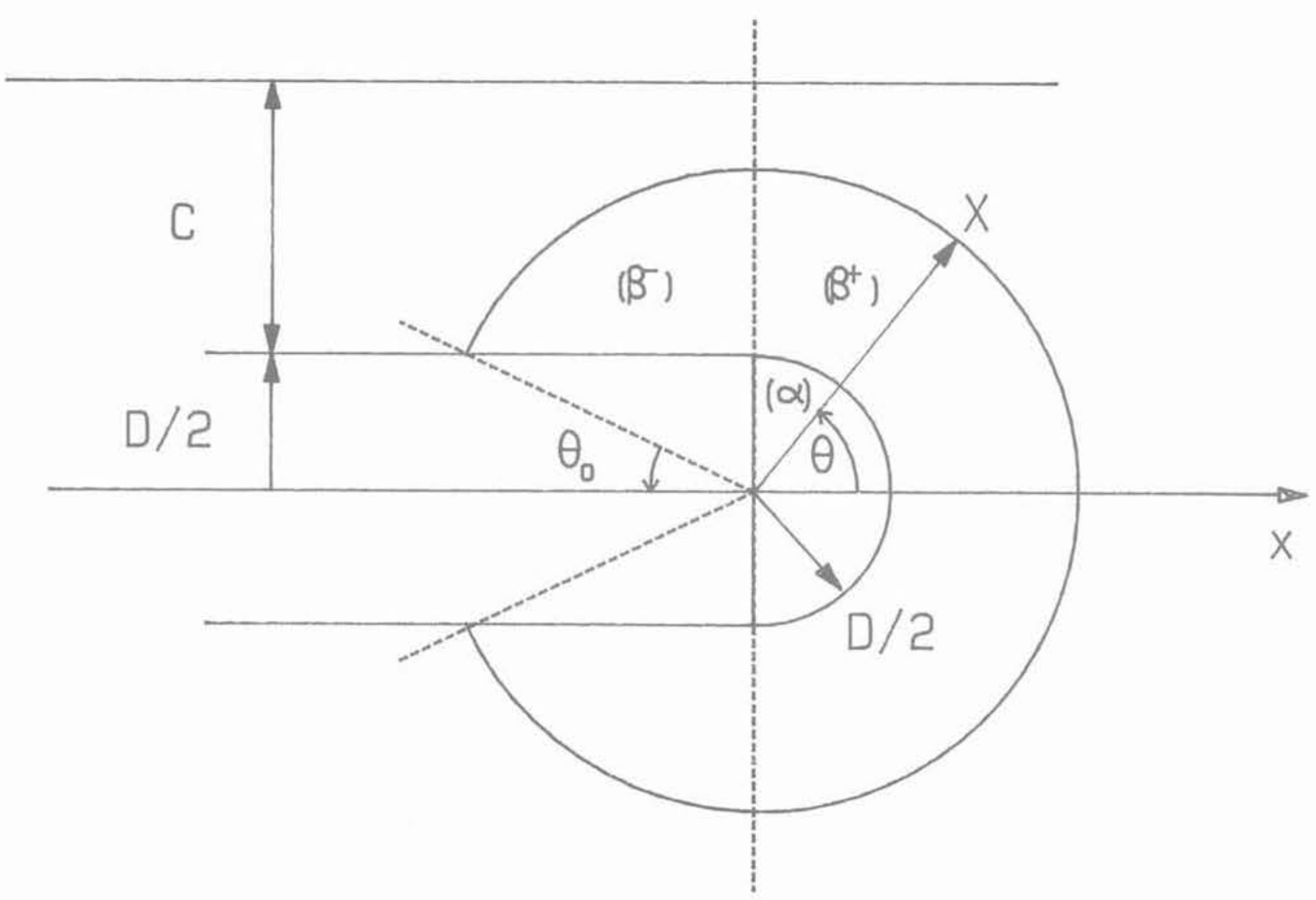

Fig. 6. - Géométrie relative au champ de contraintes 4.1.1.

Fig. 6. - Geometry relative to stress field 4.1.1.

En tenant compte de (16), il est facile de voir que la contribution du domaine $(\beta)$ vaut:

$$
\begin{aligned}
& W_{\beta}^{*}=\frac{3(1-2 \nu)}{2 E}\left[A^{2} \int_{(\beta)} d \Omega\right. \\
& \left.+\frac{2(1+\nu)}{1-2 \nu} B^{2} \int_{(\beta)}\left(1 / \mathrm{r}^{6}\right) d \Omega\right]
\end{aligned}
$$

Pour le calcul de $\mathrm{W}_{\beta}^{*}$, il est commode de scinder $(\beta)$ en deux sous-domaines situés respectivernent en avant et en arrière du front de taille :

$\left(\beta^{+}\right): r \in[R, X]: \theta \in\left[0, \frac{\pi}{2}\right] ; \varphi \in[0,2 \pi]$

$\left(\beta^{-}\right): r \in\left[\frac{\mathrm{R}}{\sin \theta}, \mathrm{X}\right] ; \theta \in\left[\frac{\pi}{2}, \pi-\theta_{0}\right] ;$

$\varphi \in[0,2 \pi]$ avec $\theta_{\mathrm{o}}(\mathrm{X})=\arcsin \left(\frac{1}{\rho_{\mathrm{X}}}\right)$

Il vient alors :

$$
\begin{aligned}
& \mathrm{W}_{\beta^{+}}=\pi \mathrm{R}^{3}\left(\delta \mathrm{p}^{2} / \mathrm{E}\right) \frac{1}{\rho_{\mathrm{x}}^{3}-1} \\
& \left(1-2 v+\rho_{\mathrm{x}}^{3} \frac{1+\nu}{2}\right)
\end{aligned}
$$

$$
\begin{aligned}
& \mathrm{W}_{\beta^{-}}^{*}=\pi \mathrm{R}^{3}\left(\delta \mathrm{p}^{2} / \mathrm{E}\right) \frac{1}{\left(\rho_{\mathrm{x}}^{3}-1\right)^{2}} \\
& \left((1-2 \nu)\left(\rho_{\mathrm{x}}^{2}-1\right)^{3 / 2}\right. \\
& +\frac{3}{16}(1+\nu)\left[\rho_{\mathrm{x}}^{6}\left(\frac{\pi}{2}-\arcsin \frac{1}{\rho_{\mathrm{x}}}\right)\right. \\
& \left.\left.+\rho_{\mathrm{x}}^{2} \sqrt{\rho_{\mathrm{x}}^{2}-1}\left(\rho_{\mathrm{x}}^{2}-2\right)\right]\right)
\end{aligned}
$$

Le calcul numérique de $\mathrm{W}^{*}\left(\underline{\underline{\sigma}}^{\prime}\right)$, c'est-à-dire la somme des contributions des domaines $(\alpha),\left(\beta^{+}\right)$et $\left(\beta^{-}\right)$indique que le minimum par rapport à $\mathrm{X}$ est atteint pour $\hat{\mathrm{X}}=\mathrm{R}+\mathrm{C}$. En normalisant le volume $\delta \mathrm{V}_{1}$ par celui de la sphère de rayon $R$, soit $V_{\text {sph }}=\frac{4}{3} \pi R^{3}$, on dispose alors d'une majoration du rapport $\delta V_{1} / V_{\text {sph }}$ de la forme:

$$
\delta \mathrm{V}_{1} / \mathrm{V}_{\text {sph }} \leq(\delta \mathrm{p} / \mathrm{E}) \operatorname{TL}(\rho, \nu)
$$

où $\rho=1+\mathrm{C} / \mathrm{R}$ et

$\Re(\rho, \nu)=\frac{3}{2} \frac{1}{\left(\rho^{3}-1\right)}$.

$$
\left((1-2 \nu)\left[\rho^{3}\left(\rho^{3}-1\right)+\left(\rho^{2}-1\right)^{3 / 2}\right]\right.
$$




$$
\begin{aligned}
& +\frac{3}{16}(1+\nu)\left[\rho^{6}\left(\frac{8}{3}+\frac{\pi}{2}-\arcsin \frac{1}{\rho}\right)\right. \\
& \left.\left.+\rho^{2} \sqrt{\rho^{2}-1}\left(\rho^{2}-2\right)-\frac{8}{3} \rho^{3}\right]\right)
\end{aligned}
$$

La représentation graphique de ce résultat est donnée à la figure 7 pour différentes valeurs de $\nu$.

\subsubsection{Champ en coordonnées cylindriques}

L'examen de la formule (23) indique que l'énergie élastique de contraintes du champ précédent tend vers l'infini quand le rapport $\rho=1+\mathrm{C} / \mathrm{R}$ tend vers 1 . Bien que le cas extrême $\rho=1$ ne soit évidemment pas rencontré dans la pratique, il est intéressant de développer une approche statique plus performante que la précédente pour les faibles valeurs de $C / R$. Une idée consiste à construire un champ de contraintes planes parallèlement au plan horizontal $\left(e_{x}, e_{z}\right)$, qui soit indépendant de la valeur de la profondeur de la couverture C. Plus précisément, en utilisant le système de coordonnées cylindriques d'axe $\mathrm{Oy}$, un tel champ $\underline{\sigma}^{\prime}$ s.a. avec $\delta p$ peut être défini de la manière suivante (fig. 8 ) :

$-\underline{\underline{a}}^{\prime}=\underline{0}$ en dehors de la région du massif contenue entre les plans $y= \pm R$;

$-\underline{\underline{\sigma}}=\delta p\left(\underline{e}_{x} \otimes \underline{e}_{x}+\underline{e}_{z} \otimes \underline{e}_{z}\right)$ dans la partie $\bar{y}$ du massif située entre les plans $y= \pm R$ et à l'intérieur du cylindre d'axe $\mathrm{Oy}$, de rayon $\mathrm{R}$;

Dans le domaine Y' défini par $\mathrm{r}>\mathrm{R},|\mathrm{y}|<\mathrm{R}$, $\sigma^{\prime}$ coïncide avec la solution en contraintes planes du problème de la cavité cylindrique de rayon $\mathrm{R}$ soumise à une traction radiale uniforme $-\delta$ p $\underline{e}_{x}$, c'est-à-dire :

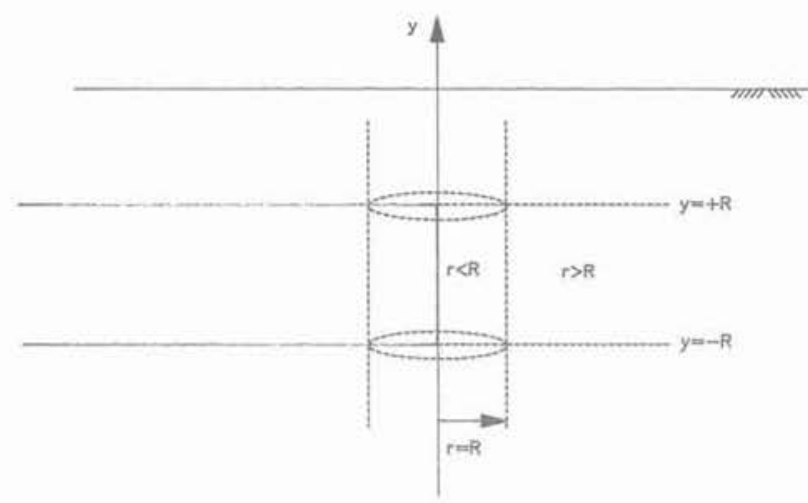

Fig. 8. - Geométrie relative au champ de contraintes 4.1.2.

Fig. 8. - Geometry relative to stress field 4.1.2.

$\sigma_{r r}^{\prime}=\delta p\left(\frac{R}{r}\right)^{2} ; \sigma_{\theta \theta}^{\prime}=-\sigma_{r}^{\prime} ; \sigma_{y y}^{\prime}=0$

Dans le domaine $Y$, la densité volumique d'énergie élastique de contraintes est constante, de même que le champ $\underline{\underline{\underline{\sigma}}}$ lui-même. Elle vaut :

$$
\begin{aligned}
& \left.\frac{1}{2}\left\{\frac{1+\nu}{\mathrm{E}} \operatorname{tr} \underline{\underline{\sigma}}^{, 2}\right)-\frac{\nu}{\mathrm{E}}\left(\operatorname{tr} \underline{\underline{\sigma^{\prime}}}\right)^{2}\right\} \\
& =\frac{\delta \mathrm{p}^{2}}{\mathrm{E}}(1-\nu)
\end{aligned}
$$

La contribution $W_{\text {yy }}$ du domaine $Y$ à l'énergie élastique de contraintes vaut donc:

$$
W_{y}=\frac{\delta p^{2}}{E}(1-\nu) \int_{y} d \Omega
$$

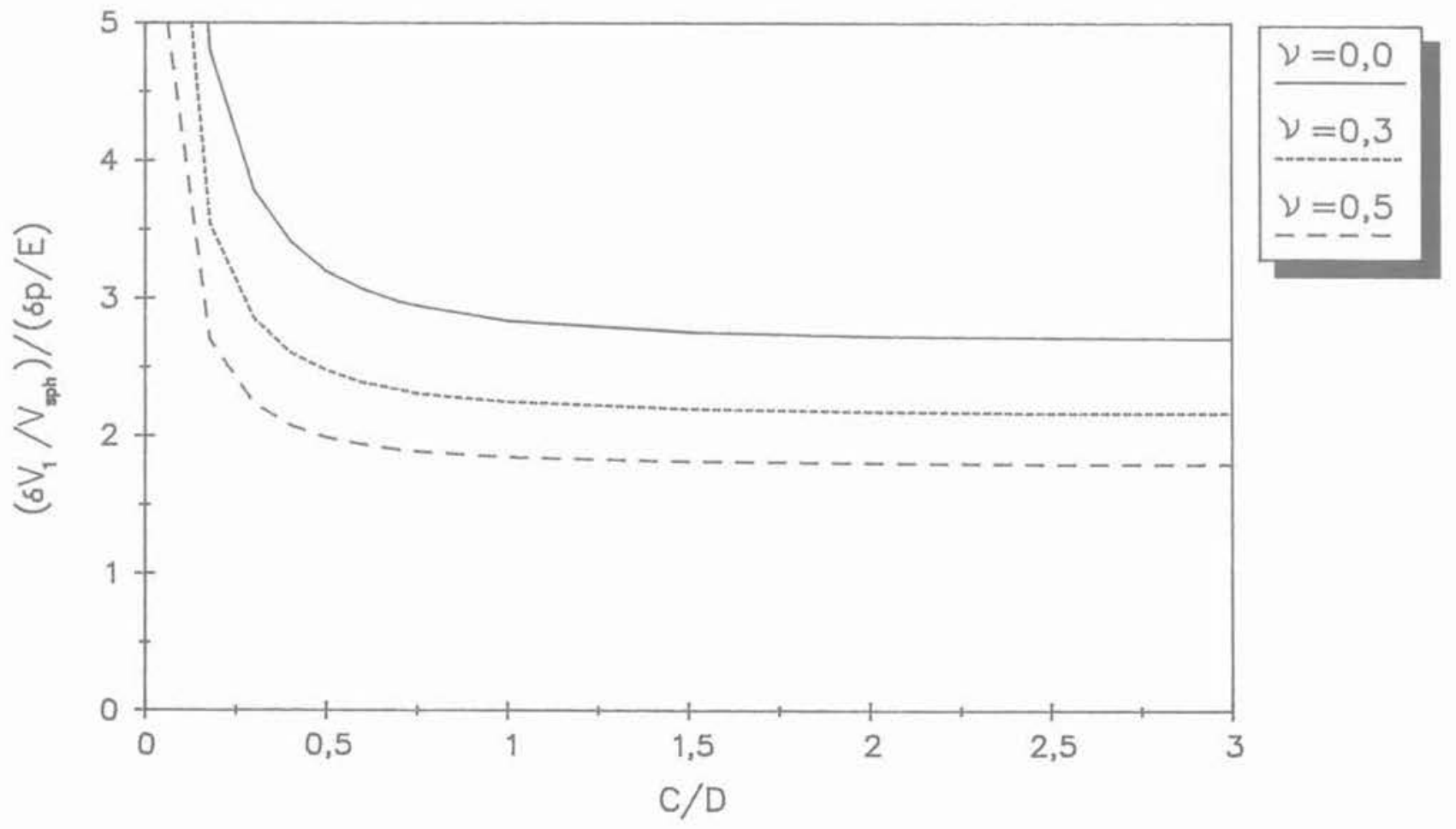

Fig. 7. - Majoration de la venue du sol au front de taille obtenue par le champ de contraintes 4.1.1.

Fig. 7. - Upper estimate of ground loss at the tunnel face obtained from stress field 4.1.1. 
Le calcul du volume de $\mathcal{Y}$ est fourni en annexe. Dans le domaine 'Y', la densité volumique d'énergie élastique de contraintes vaut $\frac{1+\nu}{\mathrm{E}} \delta \mathrm{p}^{2}\left(\frac{\mathrm{R}}{r}\right)^{4}$. L'expression de la contribution de 'Y' à $W^{*}\left(\underline{\underline{\sigma^{\prime}}}\right)$ en résulte :

$$
W_{\dot{y}} \cdot\left(\underline{\underline{\sigma^{\prime}}}\right)=\frac{1+\nu}{\mathrm{E}} \delta \mathrm{p}^{2} \mathrm{R}^{4} \int_{y^{\prime}} \mathrm{d} \Omega / \mathrm{r}^{4}
$$

Le calcul de l'intégrale intervenant dans (27) est également détaillé en annexe. Finalement, en utilisant (26) et (27), la seconde inégalité de (15) s'écrit :

$$
\begin{aligned}
& \delta \mathrm{V}_{1} / \mathrm{V}_{\mathrm{sph}} \leq(\delta \mathrm{p} / \mathrm{E})(3 / 2 \pi) \\
& \left(4 \pi-17 / 3+g+\nu\left(g-\frac{1}{3}\right)\right)
\end{aligned}
$$

où

$$
g=\int_{0}^{\pi / 2} \frac{t d t}{\sin t}
$$

On observera que la majoration obtenue est, à la différence de (22), indépendante du rapport $\mathrm{C} / \mathrm{R}$ (fig. 9). Comme on l'a annoncé, elle ne revêt d'intérêt que pour les faibles valeurs de ce rapport.

\subsection{Cas de charge $n^{\circ} 2$}

\subsubsection{Champ en coordonnées cylindriques}

Le chargement de traction - $\delta$ pe, exercé sur le massif au niveau du tunnel possède la symétrie de révolution cylindrique autour de laxe Ox. Il est naturel de compléter cette symétrie en cherchant tout d'abord à équilibrer la traction $\delta \mathrm{p}$ par un champ de contraintes nul en dehors du cylindre d'axe $\mathrm{Ox}$ de rayon $\mathrm{R}+\mathrm{C}$. Ce choix permet manifestement de respecter la condition aux limites en contraintes sur le plan $y=R+C$ (plan libre de contraintes). Il reste à construire le champ $\sigma$ ' dans la partie du massif située à l'intérieur du cylindre en question. On adopte le système de coordonnées cylindriques d'axe $\mathrm{Ox}$.

Une idée consiste à construire, dans chacun des tronçons $x<-(L+e) ; x \in]-(L+e),-e[$; $x>-e$ un état de contraintes planes parallèlement à Oyz, possédant de plus la symétrie de révolution cylindrique (fig. 10). Dans chacune de ces trois zones, le champ $\sigma$ est alors, par définition, fonction de la seule variable $r$. Par ailleurs, la condition de symétrie cylindrique $\left(\frac{\partial}{\partial \theta}(\underline{\underline{\sigma}})=\underline{\underline{0}}\right)$ combinée avec les é quations d'équilibre impose que $\underline{e}_{r}$ et $\underline{e}_{\theta}$ soient en tout point directions principales du tenseur $\underline{\sigma}^{\prime}(r)$. Enfin, la condition de contraintes planes parallèlement à Oyz assure la continuité du vecteur-contrainte $\underline{\underline{\sigma}}$. $\underline{e} \times$ (égal à 0 ) aux interfaces $\mathrm{x}=-(\mathrm{L}+e)$ et $\mathrm{x}=-e$.

Dans les tronçons d'extension infinie $x<-(L+e)$ et $x>-e$, le choix $\underline{\sigma}^{\prime}=0$ est nécessaire pour la contribution de ces domaines à l'énergie élastique de contraintes soit finie. Elle est alors évidemment nulle. $\mathrm{Ce}$ choix assure évidemment la continuité de $\underline{\sigma} \cdot \underline{e}_{r}$ à l'interface $r=R+C$. Par ailleurs, il est statiquement admissible avec la condition de front de taille libre de contraintes.

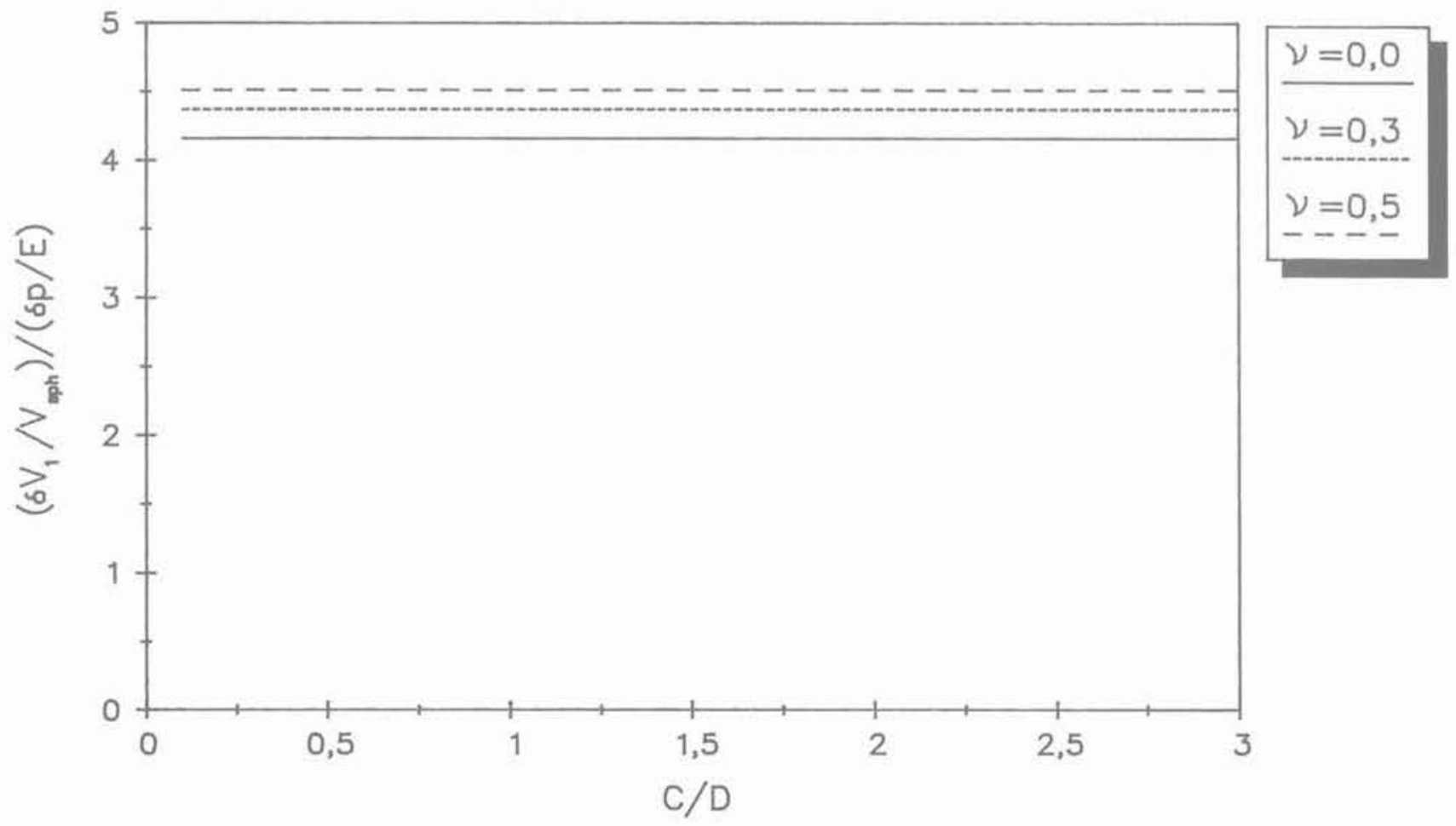

Fig. 9. - Majoration de la venue de sol au front de taille obtenue par le champ de contraintes 4.1.2. Fig. 9, - Upper estimate of ground loss at the tunnel face obtained from stress field 4.1.2. 


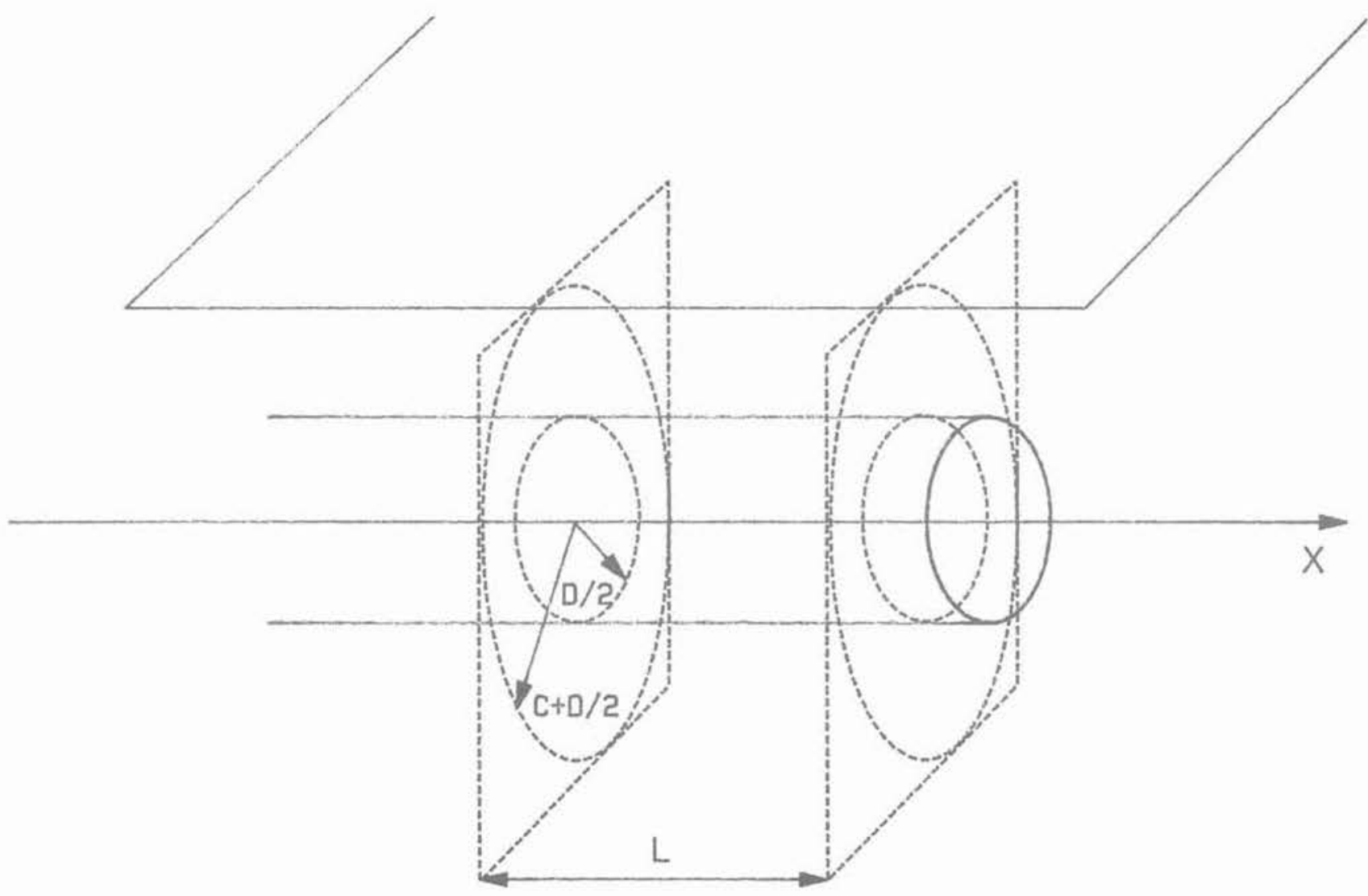

Fig. 10. - Géométrie relative au champ de contrainres 4.2.1.

Fig. 10, - Geometry relative to stress field 4.2.1.

Dans le tronçon médian $X$ défini par $\mathrm{x} \in \mathrm{J}-(\mathrm{L}+e)$, $-e[$ et $r \in] R, R+C$ [, la forme la plus générale de $\underline{\underline{\sigma}}$, dans le cadre des hypothèses de contraintes planes et de symétrie cylindrique, est la suivante:

$$
\begin{aligned}
& \underline{o}^{\prime}=f(r) \underline{e}_{r} \otimes \underline{e}_{r} \\
& +\left(r f^{\prime}(r)+f(r)\right) \underline{e}_{\theta} \otimes \underline{e}_{\theta}
\end{aligned}
$$

pour toute fonction $f(r)$ compatible avec les conditions aux limites imposées sur les cylindres $r=R$ et $r=$ $\mathrm{R}+\mathrm{C}$, c'est-à-dire :

$$
f(R)=\delta p \quad f(R+C)=0
$$

Le choix optimal de la fonction $f$ minimise la contribution du tronçon $x \in]-(L+e),-e[$ à l'énergie élastique de contraintes. En vertu du principe de minimum de l'énergie complémentaire, il correspond nécessairement à la solution en contraintes planes du problème d'un cylindre circulaire soumis à la traction $\delta p$ sur sa face interne $(r=R)$ et libre de contraintes sur sa face externe $(r=R+C)$, pour lequel on a classiquement :

$$
\begin{aligned}
& f(r)=a+b / r^{2} \quad a=-b /(R+C)^{2} \\
& b=\delta p \frac{R^{2}(R+C)^{2}}{(R+C)^{2}-R}
\end{aligned}
$$

En résumé, le champ $\underline{\underline{\sigma}}$ ainsi construit est le suivant :

$$
r>R+C: \underline{\underline{\sigma}}=\underline{\underline{0}}
$$

$$
\begin{aligned}
& \mathrm{R}<\mathrm{r}<\mathrm{R}+\mathrm{C}: \\
& \mathrm{x} \in]-(\mathrm{L}+\mathrm{e}),-e[ \\
& \left\{\begin{array}{l}
\sigma_{\pi}^{\prime}=\mathrm{a}+\mathrm{b} / \mathrm{r}^{2} \\
\sigma_{\theta \theta}^{\prime}=\mathrm{a}-\mathrm{b} / \mathrm{r}^{2} \\
\text { autres } \sigma_{\eta}^{\prime}=0
\end{array} \quad \underline{\underline{\sigma}}=\underline{\underline{0}}\right.
\end{aligned}
$$

La contribution du tronçon médian $X$ à l'énergie élastique de contraintes $W^{*}(\underline{\underline{\sigma}})$ coïncide avec $W^{*}(\underline{\underline{\underline{\sigma}}})$ et vaut :

$$
\begin{aligned}
& \left.W^{*} \underline{\underline{\underline{\sigma}}}\right)=\frac{1}{E}\left((1-\nu) \mathrm{a}^{2}\left(\int_{X} \mathrm{~d} \Omega\right)\right. \\
& \left.+(1+\nu) \mathrm{b}^{2}\left(\int_{X} \mathrm{~d} \Omega / \mathrm{r}^{4}\right)\right)
\end{aligned}
$$

soit, en posant $\rho=1+\mathrm{C} / \mathrm{R}$ :

$$
\begin{aligned}
& W^{*}(\underline{\underline{\sigma}})=\frac{(\delta \mathrm{p})^{2}}{\mathrm{E}} \pi \mathrm{R}^{2} \mathrm{~L} \\
& \left(\frac{(1+\nu) \rho^{2}+(1-\nu)}{\rho^{2}-1}\right)
\end{aligned}
$$

On observera que $\pi R^{2} L$ correspond au volume $V_{\text {exc }}$ excavé dans le déplacement du bouclier. L'inégalité de droite dans (15) fournit finalement: 


$$
\begin{aligned}
& \delta \mathrm{V}_{2} / \mathrm{V}_{\text {exc }} \leq 2(\delta \mathrm{p} / \mathrm{E}) \\
& \left(\frac{(1+\nu) \rho^{2}+(1-\nu)}{\rho^{2}-1}\right)
\end{aligned}
$$

La représentation graphique de ce résultat est donnée par les courbes de la figure 11.

\subsubsection{Champ en coordonnées sphériques}

On adopte le système de coordonnées sphériques $r, \theta$, $\varphi$ où le rayon r est compté à partir du point O' de l'axe Ox situé à mi-distance des plans $\mathrm{x}=-(\mathrm{L}+\mathrm{e})$ et $\mathrm{x}=-\mathrm{e}$ entre lesquels est appliquée la traction $\delta p$ (fig. 12). On pose $\beta=\operatorname{arctg}\left(\frac{\mathrm{L}}{2 \mathrm{R}}\right)$. On se

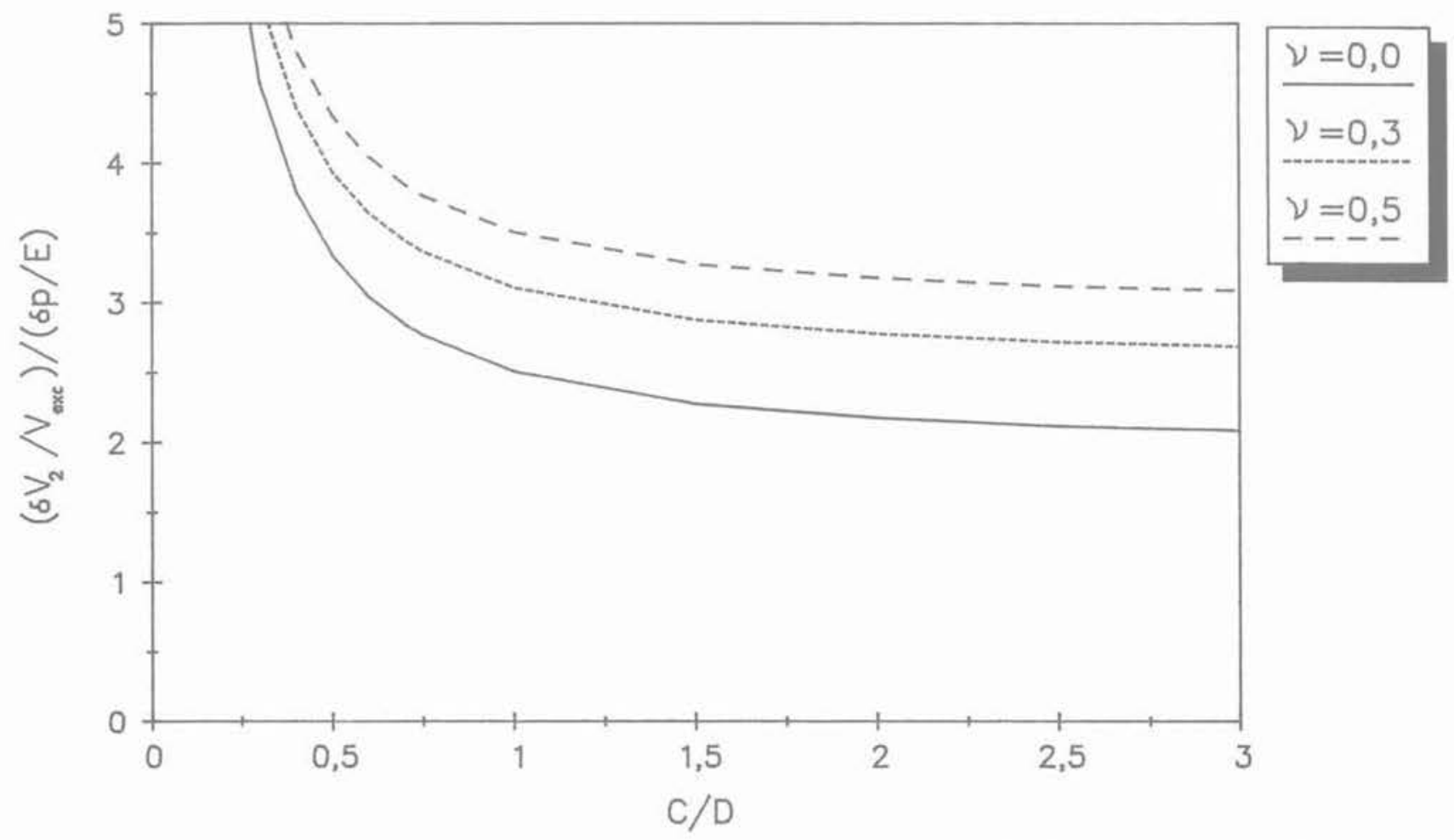

Fig. 11. - Majoration de la venue de sol à l'arrière du tunnelier obtenue par le champ de contraintes 4.2.1. Fig. 11. - Upper estimate of ground loss at the shield tail obtained from stress field 4.2.1.

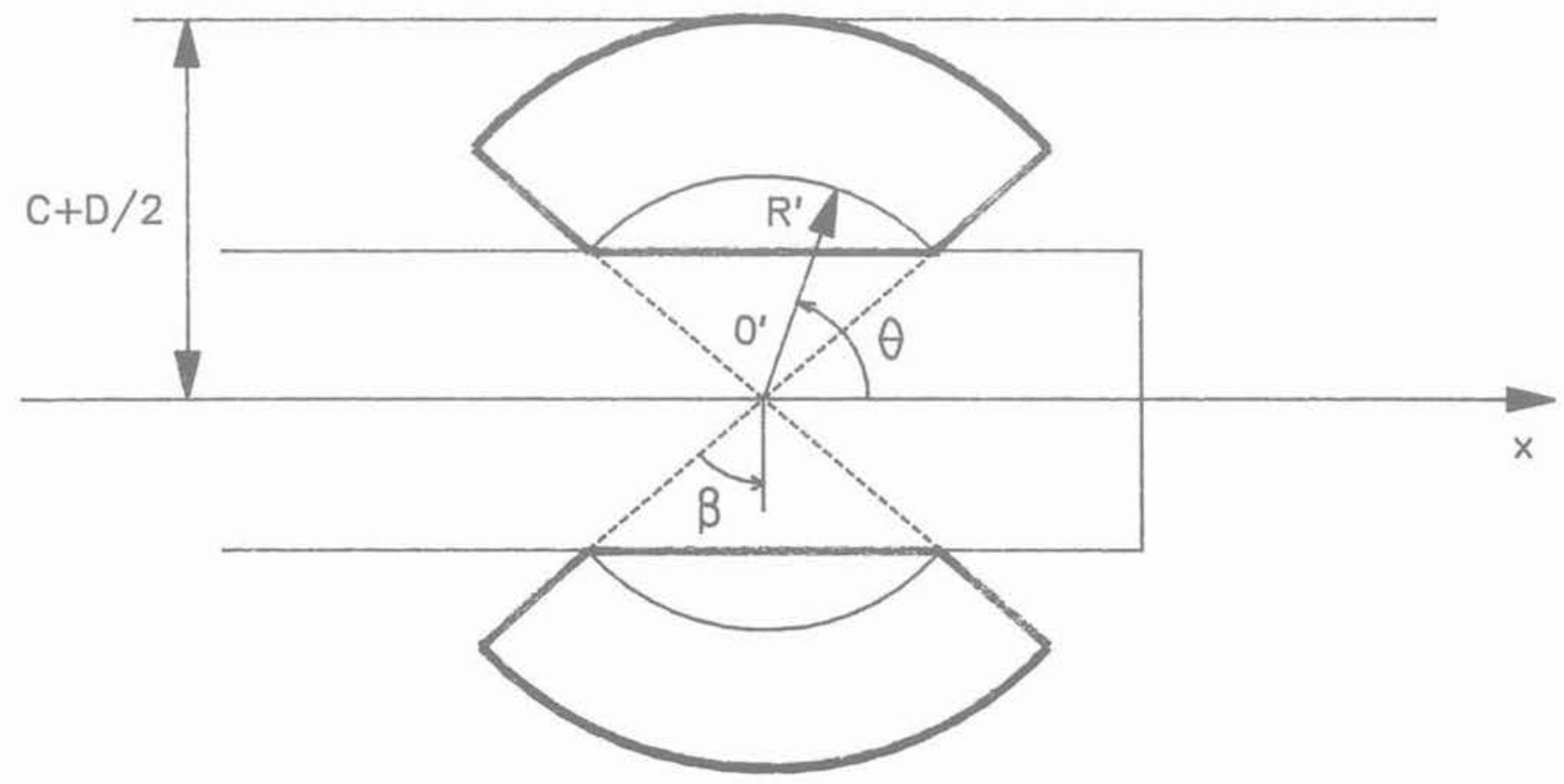

Fig. 12. - Géométrie relative au champ de contrainte 4.2.2.

Fig. 12. - Geometry relative to stress field 4.2.2. 
propose d'équilibrer le chargement par un champ de contraintes nul en dehors de la région du massif définie par $\mathrm{r} \leq \mathrm{R}+\mathrm{C}, \theta \in\left[\frac{\pi}{2}-\beta, \frac{\pi}{2}+\beta\right]$ (délimitée en traits renforcés sur la figure 12) et possédant la symétrie de révolution autour de l'axe O'x. Par rapport au champ construit en 4.2 .1 ., il s'agit qualitativement d'augmenter le volume du massif intéressé par la traction ôp afin d'améliorer la majoration (36) lorsque la géométrie du problème rend peu réaliste l'hypothèse de contraines planes. Le champ $\underline{\underline{\sigma}}$ ', indépendant de $\varphi$, est construit par morceaux de la manière suivante :

- pour $\mathrm{r} \leq \mathrm{R}^{\prime}=\mathrm{R} / \cos \beta$ (domaine $\mathcal{Z}$ ) ; le champ $\underline{\underline{\sigma}}$ est constant égal à $\delta \mathrm{p} \underline{\underline{1}}$.

- pour $r \in \mathrm{R}^{\prime}, \mathrm{R}+\overline{\mathrm{C}}$ ] (domaine $\mathcal{Z}^{\prime}$ ) : on suppose que $\underline{e}_{r}, \underline{e}_{\theta}$, et $\underline{e}_{\varphi}$ sont directions principales du tenseur $\underline{\underline{\sigma}}(\mathrm{r}, \theta)$ et que $\sigma_{\pi}$ est indépendant de $\theta$. Cette dernière condition est suggérée par la condition de continuité de $\sigma_{\pi}^{\prime}$ à l'interface $r=R^{\prime}$ entre $\mathcal{Z}$ et $\mathcal{Z}$ qui s'écrit $\sigma_{\pi}^{\prime}\left(\mathrm{R}^{\prime}\right)=\delta$ p.

La forme la plus générale d'un champ de contraintes $\underline{\sigma}$ s.a. vérifiant l'ensemble des hypothèses indiquées est la suivante :

$\sigma_{\pi}^{\prime}=f(r)$

$$
\begin{aligned}
& \sigma_{\theta \theta}=\left(\mathrm{f}(\mathrm{r})+\frac{1}{2} \mathrm{rf}(\mathrm{r})\right)\left(1-\left(\frac{\cos \beta}{\sin \theta}\right)^{2}\right) \\
& \left.\sigma_{\varphi \varphi}^{\prime}=\left(\mathrm{f}(\mathrm{r})+\frac{1}{2} \mathrm{rf}^{\prime}(\mathrm{r})\right)\left(1+\frac{\cos \beta}{\sin \theta}\right)^{2}\right)
\end{aligned}
$$

pour toute fonction $f(r)$ de classe $C^{1}$ vérifiant

$$
f\left(R^{\prime}\right)=\delta p \quad f(R+C)=0
$$

On observera toutefois que la construction proposée n'est possible que si $R^{\prime} \leq R+C$, soit :

$$
\frac{C}{R} \geq \sqrt{1+\left(\frac{L}{2 R}\right)^{2}}-1
$$

Les contributions de $\mathcal{Z}$ et $\mathcal{Z}$ ' à l'énergie élastique de contraintes sont respectivement les suivantes :

$$
W_{z}^{*}=\frac{\delta p^{2}}{2 E}(1-2 v) \mathrm{aR}^{3}
$$

et

$$
\begin{aligned}
& \mathrm{W}_{\tilde{Z}}^{*}=\frac{1}{2 \mathrm{E}} \\
& \left(\frac{1}{2}[\mathrm{~b}(1-\nu)+\mathrm{c}(1+\nu)] \int_{\mathrm{R}^{\prime}}^{\mathrm{R}+\mathrm{C}} \mathrm{r}^{4} \mathrm{f}^{2} \mathrm{dr}\right. \\
& -(1+\nu) \mathrm{c} \int_{\mathrm{R}^{\prime}}^{\mathrm{R}+\mathrm{C}} \mathrm{r}^{2} \mathrm{f}^{2} \mathrm{dr} \\
& \left.-\mathrm{p}^{2} \mathrm{R}^{3}[\mathrm{~b}(1-2 \nu)+\mathrm{c}(1+\nu)]\right)
\end{aligned}
$$

où $a, b$ et $c$ sont des fonctions sans dimension de l'angle $\beta$ :

$$
\begin{aligned}
& \mathrm{a}=4 \pi \sin ^{3} \beta \\
& \mathrm{b}=4 \pi \sin \beta \\
& \mathrm{c}=\pi \cos ^{2} \beta\left(2 \sin \beta+\cos ^{2} \beta \ln \frac{1+\sin \beta}{1-\sin \beta}\right)
\end{aligned}
$$

Le choix optimal $\tilde{f}$ de la fonction $f$ dans (37) réalise le minimum de $W^{*}\left(\underline{\underline{\sigma}}^{\prime}\right)$, c'est-à-dire celui de $W_{z}^{*}$. La détermination de $\bar{f}$ est un problème classique de calcul des variations. On montre que cette fonction est solution de l'équation différentielle :

$$
\tilde{f} " \prime+\frac{4}{r} \tilde{f},+\left(d / r^{2}\right) \tilde{f}=0
$$

et des conditions aux limites (38), où l'on a posé dans (43) :

$$
\mathrm{d}(\beta, \nu)=\frac{2(1+\nu) c}{(1+\nu) c+(1-\nu) b}
$$

Introduisant de plus les notations :

$$
\begin{aligned}
& \gamma=\frac{R^{\prime}}{R+C}=\frac{1}{\rho} \sqrt{1+\left(\frac{L}{2 R}\right)^{2}} \\
& \alpha=\frac{1}{2}(3-\sqrt{9-4 d})
\end{aligned}
$$

il est facile de voir que la solution de (43) et (38) est donnée par :

$$
\begin{aligned}
& \tilde{f}(r)=\delta p\left(\frac{1}{1-\gamma^{3-2 \alpha}}\left(\frac{\mathrm{R}^{\prime}}{\mathrm{r}}\right)^{3-\alpha}\right. \\
& \left.-\frac{\gamma^{3-2 \alpha}}{1-\gamma^{3-2 \alpha}}\left(\frac{\mathrm{R}^{\prime}}{\mathrm{r}}\right)^{\alpha}\right)
\end{aligned}
$$

Finalement, en utilisant l'expression précédente de $\tilde{f}$ dans (41), on obtient (voir annexe) une nouvelle majoration du rapport $\delta \mathrm{V}_{2} / \mathrm{V}_{\text {exc }}$ de la forme :

$$
\delta \mathrm{V}_{2} / \mathrm{V}_{\text {exc }} \leq(\delta \mathrm{p} / \mathrm{E}) \Re\left(\nu, \rho, \frac{\mathrm{L}}{\mathrm{R}}\right)
$$

qui, à la différence de (36), fait intervenir * l'élancement $\mathrm{L} / \mathrm{R}$ de la zone d'application de la traction $\delta \mathrm{p}$. Une représentation graphique en est donnée à la figure 13. (47) constitue le résultat optimal lorsque l'on explore toute la classe des champs de contraintes de (37)-(38). On rappelle cependant que son domaine de validité est délimité par la condition (39).

\section{SYNTHÈSE DES RÉSULTATS}

La figure 14 rassemble les résultats obtenus dans le cas de charge $\mathrm{n}^{\circ} 1$ au moyen du champ de contraintes en coordonnées sphériques (trais fins) et du champ de contraintes en coordonnées cylindriques (trais renforcés). Sur l'ensemble des valeurs courantes du rapport $\mathrm{C} / \mathrm{D}$, le champ en coordonnées sphériques fournit le majorant le plus faible du rapport $\delta \mathrm{V}_{1} / \mathrm{V}_{\mathrm{sph}}$. De plus, les variations de l'estimation ainsi obtenue de $\delta \mathrm{V}_{1} / \mathrm{V}_{\text {sph }}$ deviennent négligeables au-delà de la valeur $C / D=1$. Il est alors légitime de considérer que $\delta \mathrm{V}_{1} / \mathrm{V}_{\mathrm{sph}}$ ne dépend que du coefficient de Poisson. En calculant dans (23) la valeur asymptotique de $\Re$, on vérifie que cette dépendance est affine. Il est alors commode d'utiliser le diagramme de la 


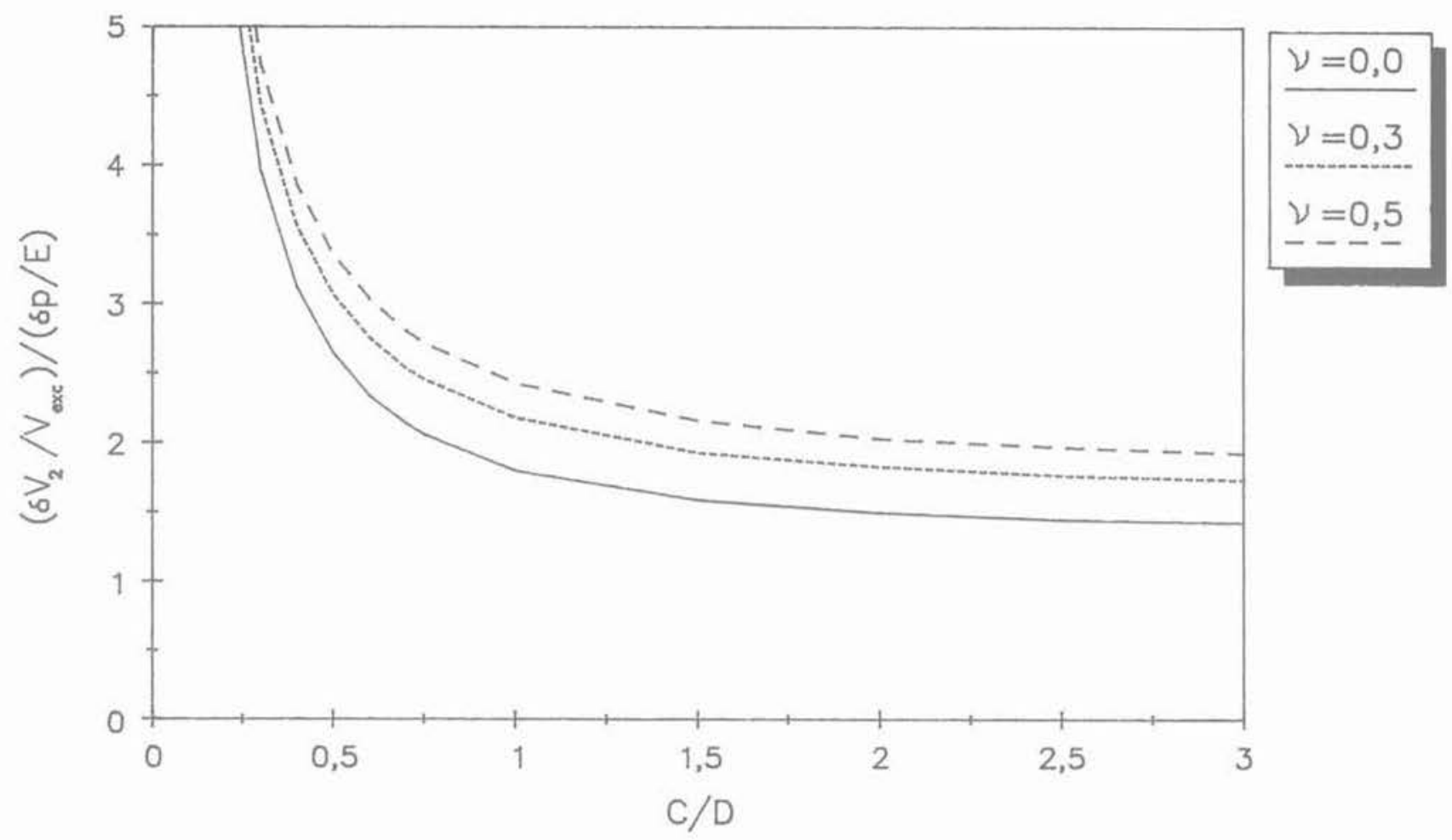

Fig. 13. - Majoration de la venue de sol à l'arrière du tunnelier obtenue par le champ de contraintes 4.2.2. Fig. 13. - Upper estimate of ground loss at the shield tail obtained from stress field 4.2.2.

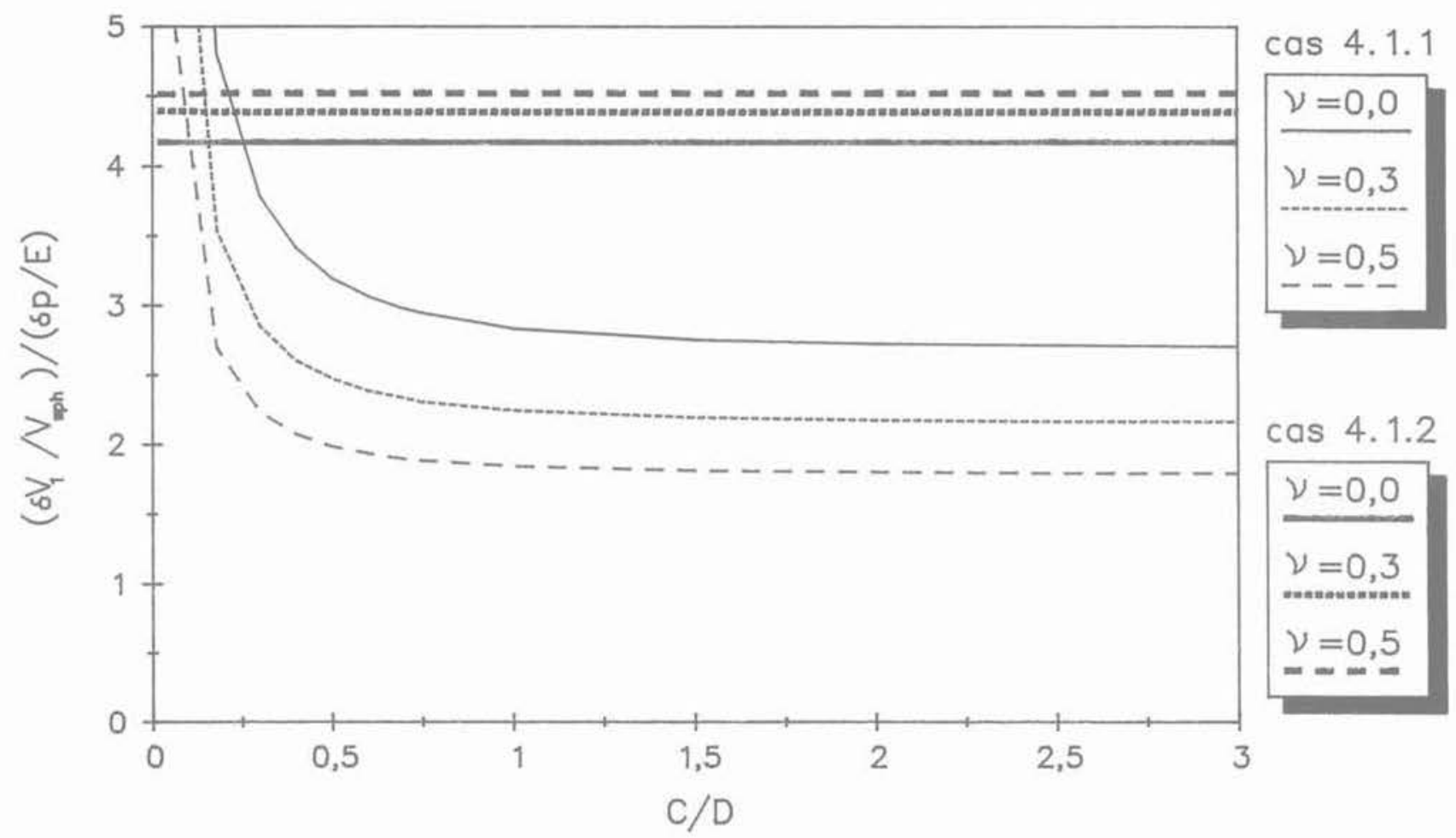

Fig. 14. - Comparaison des majorations obtenues par les champs 4.1.1. et 4.1.2.

Fig. 14. - Comparison between upper estimates obtained from stress fields 4.1.1 and 4.1.2.

figure 15 pour évaluer la venue de sol au front de taille.

Dans le domaine des faibles valeurs de C/D, l'effet de ce rapport sur la valeur du majorant de $\delta \mathrm{V}_{1} / \mathrm{V}_{\text {sph }}$ fourni par le champ sphérique doit être pris en compte. En particulier, $\mathfrak{i}$ présente une branche infinie pour la limite $C / D \rightarrow 0$. Pour les très faibles valeurs de $C / D$, c'est donc le champ en coordonnées cylindriques développé au paragraphe 4.1 .2 qui fournit le majorant pertinent pour $\delta \mathrm{V}_{1} / \mathrm{V}_{\text {sph. }}$. 


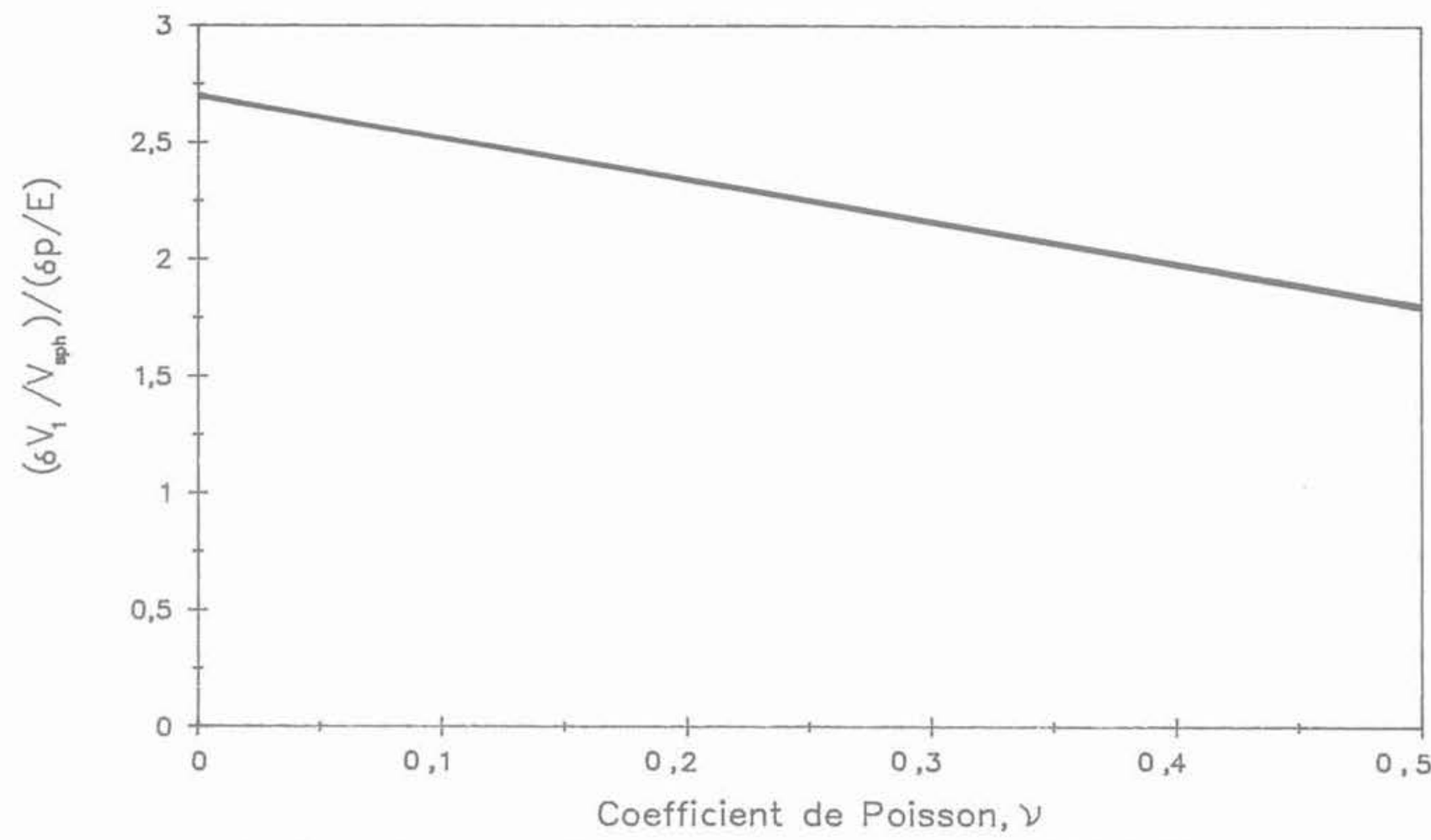

Fig. 15. - Influence du coefficient de Poisson sur la venue de sol au front de taille. Fig. 15. - Influence of the Poisson's ratio on ground loss at the tunnel face.

La figure 16 rassemble de même les résultats obtenus dans le cas de charge $n^{\circ} 2$ au moyen du champ de contraintes en coordonnées cylindriques décrit en 4.2.1. (traits fins) et du champ de contraintes en coor- données sphériques décrit en 4.2.2. (traits renforcés) pour un "élancement " $\mathrm{L} / \mathrm{R}=1 / 3$. Pour cette valeur, la condition (39) est vérifiée sur toute la gamme des variations usuelles du rapport $\mathrm{C} / \mathrm{D}$. Pour

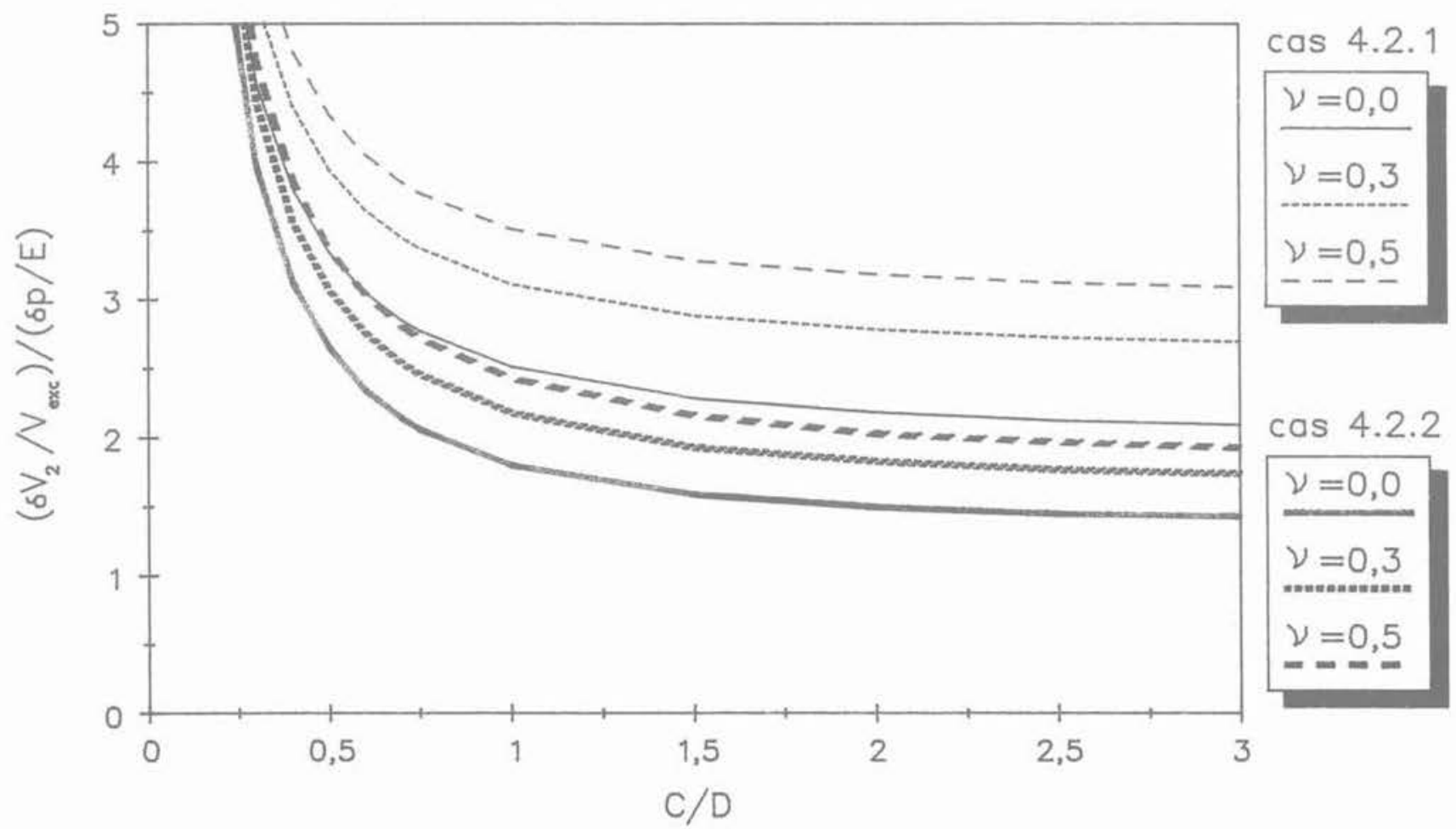

Fig. 16. - Comparaison des majorations obtenues par les champs 4.2.1 et 4.2.2.

Fig. 16. - Comparison between upper estimates obtained from stress fields 4.2.1, and 4.2.2 
toute valeur de $\nu$, le meilleur majorant est alors fourni par le champ de contraintes en coordonnées sphériques. Certes, cette conclusion, de même que les courbes en traits renforcés, dépendent a priori de l'élancement. Toutefois, la valeur $1 / 3$ adoptée pour la figure 16 correspond à l'ordre de grandeur le plus fréquemment rencontré dans la pratique. L'effet du paramètre $C / D$ apparaît plus sensible qu'à la figure 14 et ne permet pas la simplification qui avait conduit à la figure 15

\section{APPLICATION NUMÉRIQUE}

En s'appuyant sur la réflexion présentée au paragraphe 2.5., on se propose maintenant d'illustrer l'emploi des courbes des figures 14 et 16 en effectuant le calcul du tassement causé par le forage d'un tunnel avec les valeurs numériques suivantes:

$-\mathrm{H}=\mathrm{C}+\mathrm{D} / 2=15 \mathrm{~m} ; \mathrm{D}=6 \mathrm{~m} ; \mathrm{L}=1 \mathrm{~m}$; $-\gamma=20 \mathrm{kNm}^{-3} ; \mathrm{E}=60 \mathrm{MPa} ; \nu=0,5$.

Les pressions $\delta p_{1}$ et $\delta p_{2}$ à prendre en compte respectivement pour les calculs des contributions du front de taille et de la paroi latérale sont introduites sous la forme de fraction de la pression de référence $\gamma \mathrm{H}$ :

$$
\begin{aligned}
& \delta \mathrm{p}_{1}=\mathrm{x}_{1} \gamma \mathrm{H} \\
& \delta \mathrm{p}_{2}=\mathrm{x}_{2} \gamma \mathrm{H}
\end{aligned}
$$

Les figures 14 et 16 foumissent respectivement :

$$
\begin{aligned}
& \delta \mathrm{V}_{1} / \mathrm{V}_{\mathrm{sph}}=1,79\left(\delta \mathrm{p}_{1} / \mathrm{E}\right) ; \\
& \delta \mathrm{V}_{2} / \mathrm{V}_{\text {exc }}=2,02\left(\delta \mathrm{p}_{2} / \mathrm{E}\right) ;
\end{aligned}
$$

soit encore :

$$
\delta \mathrm{V} / \mathrm{V}_{\text {exc }}=\left(3,58 \mathrm{x}_{1}+1,01 \mathrm{x}_{2}\right) \%
$$

A titre indicatif, pour $\mathrm{x}_{1}=\mathrm{x}_{2}=1 / 10$, la venue de sol $\delta \mathrm{V}$ représente $0,46 \%$ du volume excavé avec une contribution prédominante du front de taille. En outre, le volume $\delta \mathrm{V}$ s'identifie au produit du volume de tassement $S_{T}$ par unité de longueur excavée par la longueur excavée L :

$$
\mathrm{S}_{\mathrm{T}}=\left(1,01 \mathrm{x}_{1}+0,28 \mathrm{x}_{2}\right) \mathrm{m}^{2}
$$

Finalement, on déduit de (5) la valeur du tassement maximal $\mathrm{s}_{\max }$ :

$$
s_{\max }=\left(0,053 x_{1}+0,015 x_{2}\right) m
$$

Si l'on fixe à nouveau $\mathrm{x}_{1}=\mathrm{x}_{2}=1 / 10$, le tassement maximal résultant du creusement est de l'ordre de $7 \mathrm{~mm}$. On obtiendrait un tassement dix fois plus élevé dans le cas d'un tunnel non revêtu et en labsence de pression au front de taille $\left(\mathrm{x}_{1}=\mathrm{x}_{2}\right.$ $=1$ ). Ces résultats soulignent la nécessité de prendre des dispositions visant à limiter les venues de sol: ajustement judicieux de la pression au front de taille, injection d'un coulis sous pression dans le vide interannulaire.

\section{CONCLUSION}

Dans le cadre de la théorie de l'élasticité, l'emploi du principe variationnel de minimum pour les contraintes a permis de majorer par voie analytique les volumes de sol débouchant dans une galerie au niveau du front de taille et à l'arrière de celui-ci. Dans le cas d'un matériau incompressible, ce travail a conduit de plus à une estimation des tassements causés par le forage d'un tunnel.

L'optimisation de la démarche passerait évidemment par une procédure numérique du type méthode des éléments finis en contraintes, dont la mise en cuvre pour une géométrie tridimensionnelle présente toutefois quelques lourdeurs.

\section{BIBLIOGRAHIE}

ATTEWELL P.B. (1977), Ground movements caused by tunneling in soil. International Conference "Large movements and structures ", Cardiff, pp. 812-948.

CLOUGH G.W., SCHMIDT B. (1981), Design and performance of excavations and tunnels in soft clays. Soft Clay Engineering, Chapitre 8, pp. 569-631, E.W. BRAND and R.P. BRENNER Eds, Elsevier Scientific Publishing Company.

CORDING E.J., HANSMIRE W.H. (1975), Displacement around soft ground tunnels. 5th Panamerican congress on soil mechanics and foundation engineering, Buenos Aires, Session IV, Tunnels in Soil, pp. 569-633.

HABIB P. (1991), Protection de l'environnement urbain par la prévention des dégâts de surface engendrés par les travaux souterrains. Proceedings of the Conference on Soil and Rock Improvement in Underground Works, Milan, 1991, vol. II, pp. 511-518.

O'REILLY M.P., NEW B.M., (1982), Settlements above tunnels in the United Kingdom; their magnitude and prediction. Tunnelling 82 , pp. 173-182.

PECK R.B. (1969), Deep excavations and tunneling in soft ground. 7th international conference on soil mechanics and foundation engineering, Stateof-the-Art Volume, pp. 225-290.

SAGASETA C., MOYA J.F., OTEO C.S. (1980), Estimation of ground subsidence over urban tunnels. 2nd International conference on ground movements and structure, Cardiff, pp. 331-344.

SALENÇON J. (1988), Mécanique des milieux continus, tome II : Elasticité - Milieux Curvilignes. Editions Ellipses.

\section{ANNEXE}

\section{Paragraphe 4.1.2.}

Le domaine Y peut être décomposé en deux volumes $\mathrm{V}^{+}$et $\mathrm{V}^{-}$selon le signe de $\mathrm{x}$ :

$$
\begin{aligned}
& x<0:\left\{\begin{array}{l}
x^{2}+z^{2} \leq R^{2} \\
y^{2}+z^{2} \geq R^{2} \\
|y| \leq R
\end{array}\right. \\
& x>0:\left\{\begin{array}{l}
x^{2}+z^{2} \leq R^{2} \\
|y| \leq R
\end{array}\right.
\end{aligned}
$$


Le volume $\mathrm{V}^{+}$, c'est-à-dire le sous-domaine correspondant à $\mathrm{x}>0$, n'est autre qu'un demi-cylindre d'axe Oy de rayon $\mathrm{R}$ et de hauteur $2 \mathrm{R}$. Le calcul de $\mathrm{V}^{+}$est donc immédiat :

$$
\mathrm{V}^{+}=\pi \mathrm{R}^{3}
$$

Le volume $\mathrm{V}^{-}$représente la partie du cylindre vertical d'axe $\mathrm{Oy}$ de rayon $\mathrm{R}$ et de hauteur $2 \mathrm{R}$ située dans le demi-espace $\mathrm{x}<0$ et à l'extérieur du cylindre horizontal d'axe $\mathrm{Ox}$ et de rayon $\mathrm{R}$. En coordonnées cartésiennes, lintégrale définissant $\mathrm{V}^{-}$n'est autre que :

$V^{-}=4 \int_{0}^{R} d y \int_{-y}^{0}\left(\sqrt{R^{2}-x^{2}}-\sqrt{R^{2}-y^{2}}\right) d x$

qui vaut :

$$
\mathrm{V}^{-}=\mathrm{R}^{3}\left(\pi-\frac{8}{3}\right)
$$

La méthode de calcul adoptée pour lintégrale intervenant dans (27) est la suivante :

$$
\int_{y^{\prime}} \mathrm{d} \Omega / \mathrm{r}^{4}=\int_{-\mathrm{R}}^{+\mathrm{R}} \mathrm{dy} \int_{\mathcal{Y}_{\mathrm{y}}} \mathrm{rdrd} \theta / \mathrm{r}^{4}
$$

où le domaine d'intégration $Y_{y}^{\prime}$ est représenté à la figure (A1). Le contour en trait renforcé qui indique la frontière intérieure de $y^{\prime}$ ' n'est autre que l'intersection d'un plan $\mathrm{y}=$ Cte avec les deux cylindres de rayon $\mathrm{R}$ respectivement d'axe $\mathrm{Ox}$ et $\mathrm{Oy}$. On observera que 'S,' évidemment symétrique par rapport au plan $z=0$, est défini en coordonnés polaires de la manière suivante :

pour $\theta \in\left[0, \pi-\theta_{y}\right] \cup\left[\pi+\theta_{y}, 2 \pi\right]: r \geq R$ pour $\theta \in\left[\pi-\theta_{y}, \pi+\theta_{y}\right]: r \geq r_{y}=R\left(\sin \theta_{y} / \sin \theta\right)$
Il vient donc :

$$
\begin{aligned}
& \int_{Y_{y}^{\prime}} \operatorname{rdrd} \theta / \mathrm{r}^{4}=2\left(\int_{0}^{\pi-\theta_{y}} d \theta \int_{R}^{+\infty} \mathrm{dr} / \mathrm{r}^{3}\right. \\
& \left.+\int_{0}^{\theta_{y}}\left(\int_{r_{y}}^{+\infty} d r / \mathrm{r}^{3}\right) d y\right)
\end{aligned}
$$

soit :

$$
\begin{aligned}
& \int_{\mathcal{Y}^{\prime}} \mathrm{d} \Omega / \mathrm{r}^{4} \\
& =2 \int_{0}^{\mathrm{R}}\left(\pi-\theta_{y}+\frac{\theta_{y}-\sin \theta_{y} \cos \theta_{y}}{2 \sin ^{2} \theta_{y}}\right) d y
\end{aligned}
$$

et finalement :

$$
\begin{aligned}
& \int_{y^{\prime}} \mathrm{d} \Omega / \mathrm{r}^{4} \\
& =\frac{1}{\mathrm{R}}\left(2 \pi-3+\int_{0}^{\pi / 2} \frac{\mathrm{tdt}}{\sin \mathrm{t}}\right)
\end{aligned}
$$

\section{Paragraphe 4.2.2.}

Le principe de calcul de $\tilde{\mathrm{f}}$ consiste à observer que la dérivée de la fonction $\tilde{\mathrm{W}}_{\mathrm{g}}(\mathrm{x})$ de la variable réelle $\mathrm{x}$ :

$$
\left.x \rightarrow W^{*} \tilde{(f}+x g\right)
$$

est nulle en $x=0$, pour tout choix d'une fonction $g$ s'annulant en $R^{\prime}$ et $R+C$. En effet, la fonction $\tilde{f}+x g$ définit alors par (37) un champ de con-

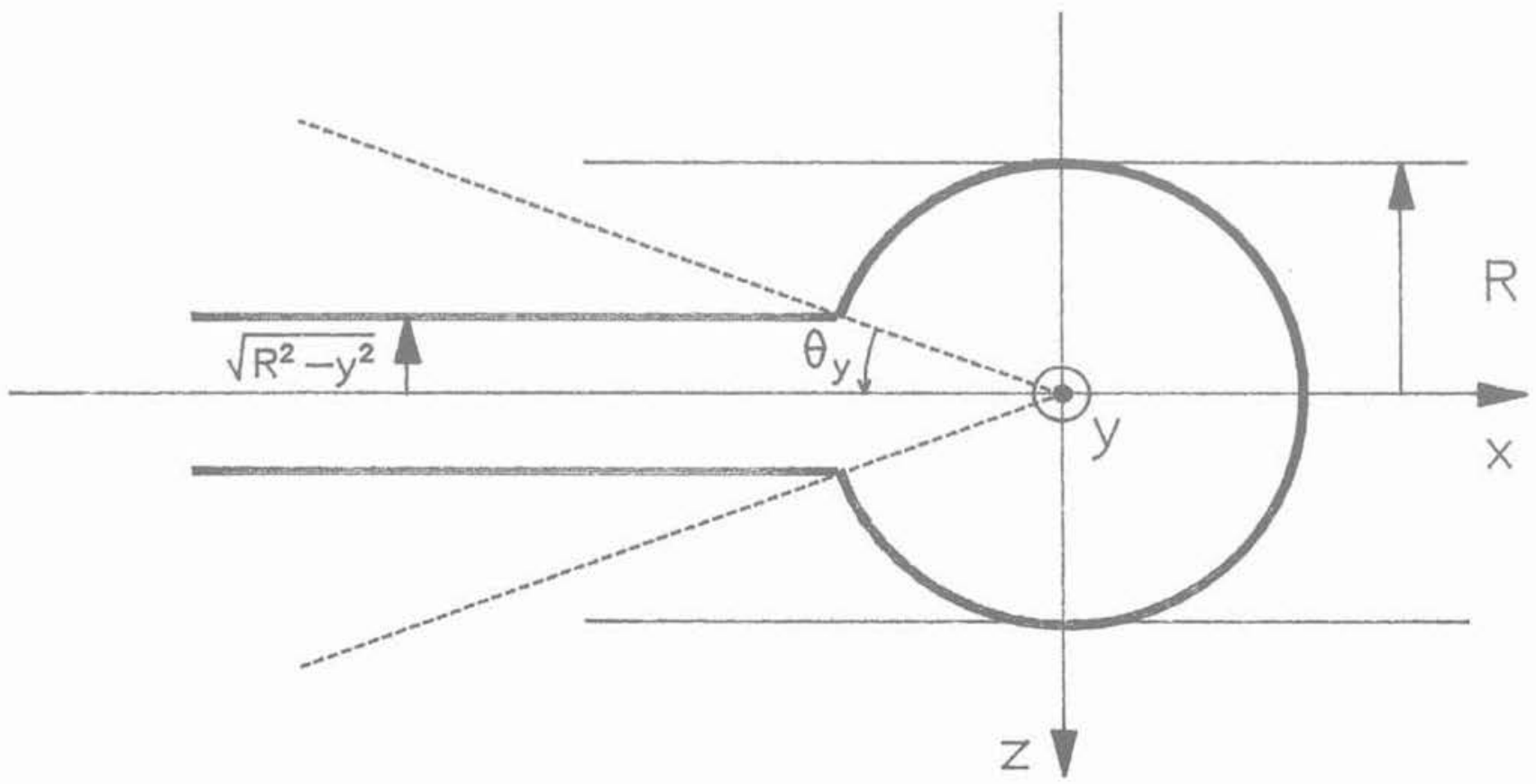

Fig. A1. - Section par un plan $y=$ Constante de la géométrie relative au champ de contraintes 4.1.2

Fig. A1. - Horizontal section of the geometry relative to stress field 4.1.2. 
traintes statiquement admissible avec $\delta \mathrm{p}$. En vertu du principe de minimum de l'énergie complémentaire, $\widetilde{\mathrm{W}}_{\mathrm{g}}(\mathrm{x})$ admet donc un minimum absolu en $\mathrm{x}=0$. La relation $\bar{W}_{\mathrm{g}}^{\prime}(0)=0$ s'exprime par:

$\int_{R^{\prime}}^{R+C} g\left(f^{\prime \prime}+\frac{4}{r} f^{\prime}+\left(d / r^{2}\right) f\right) d u=0$

Il reste à exploiter que le choix de la fonction $\mathrm{g}$ à support dans ]R', R+Cl est arbitraire pour obtenir l'équation différentielle (43).

Les expressions des intégrales intervenant dans $W_{\mathcal{Z}}^{*}$, pour loptimum $\tilde{f}$ sont les suivantes :

$$
\begin{aligned}
& \int_{R^{\prime}}^{R+C} r^{2} \tilde{f^{2}} d r=\delta p^{2} R^{\prime 3} F_{0} \\
& \int_{R^{\prime}}^{R+C} r^{4} \tilde{f}^{\prime 2} d r=\delta p^{2} R^{\prime 3} F_{1}
\end{aligned}
$$

où l'on a posé :

$$
\mathfrak{F}_{0}=\frac{1}{3-2 \alpha} \frac{1+\gamma^{3-2 \alpha}}{1-\gamma^{3-2 \alpha}}
$$

$$
+2 \ln \gamma \frac{\gamma^{3-2 \alpha}}{\left(1-\gamma^{3-2 \alpha}\right)^{2}}
$$

et :

$$
\begin{aligned}
& \digamma_{1}=\frac{1}{3-2 \alpha} \frac{(3-\alpha)^{2}+\alpha^{2} \gamma^{3-2 \alpha}}{1-\gamma^{3-2 \alpha}} \\
& +2 \alpha(3-\alpha) \ln \gamma \frac{\gamma^{3-2 \alpha}}{\left(1-\gamma^{3-2 \alpha}\right)^{2}}
\end{aligned}
$$

L'expression de la fonction $\Re$ est donc la suivante :

$$
\begin{aligned}
& \Re=\frac{1}{\pi} \frac{\mathrm{R}}{\mathrm{L}}\left(1+\left(\frac{\mathrm{L}}{2 \mathrm{~L}}\right)^{2}\right)^{3 / 2} \\
& \left(\mathrm{~b}\left[\frac{1-\nu}{2} \mathfrak{F}_{1}-\frac{1-2 \nu}{1+\left(\frac{\mathrm{L}}{2 \mathrm{R}}\right)^{2}}\right]\right. \\
& \left.+ \text { c }(1+\nu)\left[\frac{1}{2} \mathfrak{F}_{1}-\mathfrak{F}_{0}-1\right]\right)
\end{aligned}
$$

